\title{
DIFFERENTIAL OPERATOR TECHNIQUE IN THE ISING SPIN SYSTEMS
}

\author{
T. KANEYOSHI \\ Department of Physics, Nagoya University, 464-01, Nagoya, Japan
}

(Received December 17, 1992)

\begin{abstract}
A revicw is given of the differential operator technique in the Ising spin systems. The theoretical frameworks of the various models are discussed on the basis of the Ising spin identities. These can be applied to examine the magnetic properties in a variety of magnetic materials.
\end{abstract}

PACS numbers: 05.50.+q, 75.10.-b

\section{Introduction}

The Ising model and its various variants are of the most extensively studied many-body systems. The reason is due to the fact that they can describe fairly well numerous physical systems, such as magnetic spin systems, binary alloys, lattice gas, and so on.

The simplest form of the Ising model appears in one-dimensional lattice (lincar chain) consisting of spin- $1 / 2$ atoms, with nearest-neighbor interactions and in the absence of an external field. It was in this form that Ising proposed his model in 1925, in order to study the magnetic phase transition. Ilowever, he did not find a long-range order at any finite temperature. Indeed, one may say that the Ising chain undergoes a phase transition at zero temperature. Ilowever, the two-dimensional (square lattice) Ising model in the absence of an external ficld does show a phase transition at a finite temperature, which was solved exactly by Onsager in 1944. After Onsager's solution, the Ising model has been one of the most actively studied problems in statistical mechanics. Some rigorous solutions have been given for the simple Ising model of one-dimensional and certain two-dimensional lattices. There are also many results based on the scries-cxpansion and renormalization-group methods, especially for the critical region of the model. Much effort has gone into this field, but we do not yet have detailed theoretical knowledge of the three-dimensional Ising model and its variations.

On the other hand, because of its simplicity, the mean-field approximation (MFA) has played an important role for the description of cooperative phenomena, in which the effect of the ordering interactions is naively represented by that of a 
mean field proportional to the average net magnetic moment of a magnetic system. The theory can be relied on for an approximate description of the major aspects of the phenomona being studied. However, the MFA has some deficiencies, due to neglection of correlations, when MFA results are compared with experiments. Improvements in this respect have been sought by many methods.

In this work, a useful scheme for obtaining approximate solutions in the two- or more dimensional Ising systems is reviewed. The method is based on the differential operator technique introduced into exact (or approximate) Ising spin identities and is of a relatively simple mathematical character. Using this scheme we can write the identities in forms which are particularly amenable to approximation. The approximate formulation can systematically include the effects of correlations. The approach can be applied to a great variety of physical systcms, such as pure and disordered magnetic systems as well as special magnetic materials (or surfaces, thin films and multilayers) being now in current topics of magnetism, and gives an appropriate description of a system being studied. In the following sections, we shall discuss how the differential operator technique can be introduced into various types of the Ising models, and also clarify the backgrounds of the approximate formulations.

\section{Ising model and Callen identity}

The Ising model of ferromagnetism is a model whereby, because of an extreme field of anisotropy, only the $z$ component of a spin exists. The Hamiltonian of the model, in an external field $H$, is given by

$$
H=-\frac{1}{2} \sum_{i, j} J_{i j} \mu_{i} \mu_{j}-H \sum_{i} \mu_{i},
$$

where the sums run $N$ identical spins. $\mu_{i}$ is the dynamical variable which can take two values, \pm 1 , and $J_{i j}$ - the exchange interaction between a site $i$ and a site $j$. That is to say, $\mu_{i}$ is the $z$ component of a spin operator $\left(S_{i}^{z}=(1 / 2) \mu_{i}\right)$ associated with the ion localized at the site $i$ which can take spin up $\left(\mu_{i}=+1\right)$ or down $\left(\mu_{i}=-1\right)$. The spin system is ordered when all spins are up (or down) in a ferromagnet $\left(J_{i j}>0.0\right)$. The magnetic field is added in order to break the symmetry and favor the ordered phase to be up or down. The parameter that measures the ordering of the system (or the long-range order parameter) is given by $m=\left\langle\mu_{i}\right\rangle$. In the ordered phase $m \neq 0.0$, while in the disordered phase $m=0.0$.

The expectation value of the spin variable at the site $i$ is given by

$$
\left\langle m_{i}\right\rangle=\frac{1}{Z} \operatorname{Tr} \mu_{i} \mathrm{e}^{-\beta H}
$$

with

$$
Z=\operatorname{Tr} \mathrm{e}^{-\beta H},
$$

where $\operatorname{Tr}$ means the sum over allowed states of the system. Here, $\beta=1 / k_{\mathrm{B}} T$, where $k_{\mathrm{B}}$ is the Boltzmann constant and $T$ - the absolute temperature.

We know now that an exact relation can be derived for the expectation value (2), when the Hamiltonian is given by (1). For the derivation, let us separate the 
IIamiltonian (1) into two parts; one (denoted by $H_{i}$ ) which includes all contributions associated with the site $i$, and the other (denoted by $H^{\prime}$ ) which does not depend on the site $i$. Then, one has

$$
H=H_{i}+H^{\prime}
$$

with

$$
H_{i}=-\mu_{i} E_{i}
$$

and

$$
E_{i}=\sum_{i, j} J_{i j} \mu_{j}+H
$$

where $E_{i}$ is the operator expressing the local field on the site $i$. Here, notice that the spin variables commute, i.e. $\left[\mu_{i}, \mu_{j}\right]=0$, and hence

$$
\left[H_{i}, H^{\prime}\right]=\left[H_{i}, H\right]=0
$$

in the Ising model.

Because of the commutative relation, the expectation value (2) can be expressed as

$$
\left\langle\mu_{i}\right\rangle=\frac{1}{Z}\left\{\operatorname{Tr}^{-\beta H}\left[\frac{\operatorname{tr}_{(i)} \mu_{i} \exp \left(-\beta H_{i}\right)}{\operatorname{tr}_{(i)} \exp \left(-\beta H_{i}\right)}\right]\right\},
$$

where $\operatorname{tr}_{(i)}=\sum_{\mu_{i}=-1}^{+1}$ stands for the trace associated with the variable at the site $i$. By doing the partial trace of $\mu_{i}$, one obtains

$$
\left\langle\mu_{i}\right\rangle=\frac{1}{Z} \operatorname{Tr}\left[\mathrm{e}^{-\beta H} \tanh \left(\beta E_{i}\right)\right]
$$

or

$$
\left\langle\mu_{i}\right\rangle=\left\langle\tanh \left(\beta E_{i}\right)\right\rangle
$$

This is the identity first derived by Callen in 1963 [1].

By exlending the above procedure, the identity can be easily generalized to

$$
\left\langle\left\{f_{i}\right\} \mu_{i}\right\rangle=\left\langle\left\{f_{i}\right\} \tanh \left(\beta E_{i}\right)\right\rangle \text {, }
$$

where $\left\{f_{i}\right\}$ can take any function of the Ising variables as long as it is not a function of the site $i$.

Furthermore, the above derivation of (9) can be also generalized to the Ising model with a general spin $S$ expressed by

$$
H=-\frac{1}{2} \sum_{i, j} J_{i j} S_{i}^{z} S_{i}^{z}-H \sum_{i} S_{i}^{z}
$$

where $S_{i}^{z}$ takes the $(2 S+1)$ components allowed for a spin value $S$. Then, one obtains

$$
\left\langle\left\{f_{i}\right\} S_{i}^{z}\right\rangle=S\left\langle\left\{f_{i}\right\} B_{S}\left(\beta E_{i}\right)\right\rangle
$$

with

$$
E_{i}=\sum J_{i j} S_{j}^{z}+H
$$

where $B_{S}(x)$ is the Brillouin function [2]. 
At this place, notice that the standard mean-field theory can be obtained from (9) (or (12)) by approximating the thermal average of the hyperbolic tangent (or the Brillouin function) with the thermal average of $E_{i}$, i.e.,

$$
\left\langle\tanh \left(\beta E_{i}\right)\right\rangle \approx \tanh \left(\beta\left\langle E_{i}\right\rangle\right)
$$

or

$$
\left\langle B_{S}\left(\beta E_{i}\right)\right\rangle \approx B_{S}\left(\beta\left\langle E_{i}\right\rangle\right) .
$$

Thus, the exact identities (9), (10) and (12) give a way to improve the mean-field approximation.

\section{Differential operator technique}

The identities of Sec. 2 have been derived about 30 years ago. At this time, only a few authors had directed their attention to these identities for discussing the thermodynamic properties of the Ising model. During the last decade, however, numerous publications dealing with Ising systems have appeared, employing an effective-field theory which correctly accounts for the single-site kinematic relations between the spin operators. The starting point of these works is based on a set of formal identities, of the type discussed in Sec. 2.

The first systematic approach was introduced by Matsudaira [3]. In order to treat the Callen identity (9), he noticed the following exact relations valid for $\mu_{i}= \pm 1$ :

$$
\begin{aligned}
& \tanh \left(K \mu_{1}\right)=A \mu_{1}, \quad A=\tanh K, \quad \tanh \left[K\left(\mu_{1}+\mu_{2}\right)\right]=B\left(\mu_{1}+\mu_{2}\right), \\
& B=\frac{1}{2} \tanh 2 K, \quad \tanh \left[K\left(\mu_{1}+\mu_{2}+\mu_{3}\right)\right]=C_{1}\left(\mu_{1}+\mu_{2}+\mu_{3}\right)+C_{2} \mu_{1} \mu_{2} \mu_{3}, \\
& C_{1}=\frac{1}{4}(\tanh 3 K+\tanh K), \quad C_{2}=\frac{1}{4}(\tanh 3 K-\tanh K),
\end{aligned}
$$

and so on, where $K=\beta J$ for nearest-neighbor interaction $J$. For instance, the identity (9) for the honeycomb lattice with coordination number $z=3$ can be, upon using the exact relation (15), rewritten as

$$
\left\langle\mu_{i}\right\rangle=C_{1}\left(\left\langle\mu_{i+1}\right\rangle+\left\langle\mu_{i+2}\right\rangle+\left\langle\mu_{i+3}\right\rangle\right)+C_{2}\left\langle\mu_{i+1} \mu_{i+2} \mu_{i+3}\right\rangle
$$

where $i+\delta(\delta=1,2,3)$ denote the nearest-neighbors of the site $i$. However, when $E_{i}$ in (9) includes a number of Ising spins, it is not so easy to write the corresponding exact relation. Furthermore, for higher spin $(S>1 / 2)$ systems as well as random spin- $1 / 2$ systems, it is a difficult task to find such exact relations.

As is understood from (16) (or (15)), the use of the kinematic relations for the spin operators is a crucial step in the theory based on the identity (9) (or (12)) as an a verage over a finite polynomial spin operators belonging to the neighboring sites. This can be systematically and easily achieved by the use of a differential operator technique introduced by Honmura and Kaneyoshi [4]:

for $(9)$ or

$$
\tanh \left(\beta E_{i}\right)=\left.\exp \left(E_{i} \nabla\right) \tanh x\right|_{x=0}
$$

$$
B_{S}\left(\beta E_{i}\right)=\left.\exp \left(E_{i} \nabla\right) B_{S}(x)\right|_{x=0}
$$


for (12), where $\nabla=\partial / \partial x$ is a differential operator. Here, we used a mathematical relation

$$
\exp (a \nabla) \varphi(x)=\varphi(x+a) .
$$

In the following parts, let us examine at first the simplest case of $\mu_{i}= \pm 1(S=1 / 2)$. For the case of an arbitrary spin $(S>1 / 2)$, see Secs. 6 and 7 .

Noticing that

$$
\mathrm{e}^{a \mu_{i}}=\cosh a+\mu_{i} \sinh a
$$

Eq. (17) can be written as, for $H=0.0$,

$$
\tanh \left(\beta J \sum_{\delta} \mu_{i+\delta}\right)=\left.\prod_{\delta=1}^{z}\left[\cosh (J \nabla)+\mu_{i+\delta} \sinh (J \nabla)\right] \tanh x\right|_{x=0} .
$$

Here, when $z=1,2$ or 3 , the same exact relations as those of (15) can be easily derived. For example, when $z=2$,

$$
\begin{aligned}
\tanh & {\left[K\left(\mu_{i+1}+\mu_{i+2}\right)\right]=\left[\cosh (J \nabla)+\mu_{i+1} \sinh (J \nabla)\right] } \\
\times & {\left.\left[\cosh (J \nabla)+\mu_{i+2} \sinh (J \nabla)\right] \tanh x\right|_{x=0} } \\
= & \left.\left(\mu_{i+1}+\mu_{i+2}\right) \sinh (J \nabla) \cosh (J \nabla) \tanh x\right|_{x=0} \\
= & \left.\frac{1}{4}\left(\mu_{i+1}+\mu_{i+2}\right)\left(\mathrm{e}^{2 J \nabla}-\mathrm{e}^{-2 J \nabla}\right) \tanh x\right|_{x=0}=B\left(\mu_{i+1}+\mu_{i+2}\right) .
\end{aligned}
$$

Here, going from the second line to the third line in (22), we used the fact that even functions of $\nabla$ must be zero when operating to the odd function (or $\tanh x$ ). In this way, the exact relation (10) can be generally rewritten as

$$
\begin{aligned}
& \left\langle\left\{f_{i}\right\} \mu_{i}\right\rangle=\left.\left\langle\left\{f_{i}\right\} \mathrm{e}^{E_{i} \nabla}\right\rangle \tanh (\beta x)\right|_{x=0} \\
& =\left.\left\langle\left\{f_{i}\right\} \prod_{j}\left[\cosh \left(J_{i j} \nabla\right)+\mu_{j} \sinh \left(J_{i j} \nabla\right)\right]\right\rangle \tanh (\beta x+h)\right|_{x=0},
\end{aligned}
$$

where $h=\beta H$. This is also ciact and is valid for any lattice structure of a spin-1/2 Ising model. Equation (23) can generate many kinds of identities for spin correlation functions, upon substituting appropriate Ising variable functions for $\left\{f_{i}\right\}$.

For the latter discussion, let us via Eq. (23) examine the spin correlation function of the spin-1/2 linear chain with nearest-neighbor interaction $J$ [5]. Putting $\left\{f_{i}\right\}=\mu_{k}(k \neq i)$ and $H=0.0$ into (23), it gives

$$
\left\langle\mu_{k} \mu_{i}\right\rangle=\frac{1}{2} \tanh (2 \beta J)\left(\left\langle\mu_{k} \mu_{i-1}\right\rangle+\left\langle\mu_{k} \mu_{i+1}\right\rangle\right) .
$$

At this place, due to translational invariance, the correlation function $\left\langle\mu_{k} \mu_{i}\right\rangle$ depends only on the distance between $i$ and $k$ :

$$
\left\langle\mu_{k} \mu_{i}\right\rangle=\left\langle\mu_{0} \mu_{i-k}\right\rangle=\left\langle\mu_{0} \mu_{r}\right\rangle=g(r)
$$

where $r=i-k$ is a measure of the distance between spins, in units of a lattice constant. Using (25), Eq. (24) can be written as

$$
2 \operatorname{coth}(2 \beta J)=\frac{g(r+1)}{g(r)}+\left[\frac{g(r)}{g(r-1)}\right]^{-1},
$$


which implies that the right-hand side must be independent of $r$. Assuming that

$$
\frac{g(r+1)}{g(r)}=\frac{g(r)}{g(r-1)}=\gamma
$$

and taking the physically acceptable solution, the solution of (26) is given by

$$
\gamma=\tanh (\beta J)
$$

Thus, one obtains

$$
g(r)=g_{i-k}=[\tanh (\beta J)]^{r} .
$$

This is a well-known exact result for the Ising chain.

\section{Effective-field theories}

It is well known in statistical mechanics that the one-dimensional nearest-neighbor Ising model can be solved exactly. The exact solutions of the thermodynamic properties in the spin-1/2 Ising linear chain can be also derived by the use of the differential operator technique based on the exact identities. As guides to real (two- or three-dimensional) systems, however, such a model has a serious disadvantage, since it does not have a phase transition at a non-zero temperature.

On the other hand, the first step in the interpretation of the magnetic properties of a solid is usually the application of an effective-field theory. When used correctly, the theory can be relied on for an approximate description of the major aspects of the phenomena being studied. It acts as a guidepost, as it was, indicating the direction of more elaborate theoretical contractions and of more detailed experiments. In this section, let us discuss how the approximate formulations (or effective-field theories) superior to the mean-field approximation (14) can be derived systematically from the present formulation based on the Ising spin identities.

\subsection{Decoupling (or Zernike) approximation}

As is understood from (16), the right-hand side of (23) contains thermal averages of multiple correlation functions. To proceed further, one has to make some approximations, in order to treat the identities approximately. The simplest approximation, and the one most frequently adopted, is to decouple these according to

$$
\left\langle\mu_{j} \mu_{k} \ldots \mu_{l}\right\rangle \approx\left\langle\mu_{j}\right\rangle\left\langle\mu_{k}\right\rangle \ldots\left\langle\mu_{l}\right\rangle
$$

for $j \neq k \neq \ldots \neq l$.

Introducing the approximation (30), the averaged value of $\mu_{i}((23)$ with $\left.\left\{f_{i}\right\}=1.0\right)$ can be written in a compact form [6]:

$$
\left\langle\mu_{i}\right\rangle=\left.\prod_{j}\left[\cosh \left(J_{i j} \nabla\right)+\left\langle\mu_{j}\right\rangle \sinh \left(J_{i j} \nabla\right)\right] \tanh (\beta x+h)\right|_{x=0} .
$$

To simplify the notation, let us consider the case of zero-field and nearest-neighbor interactions. For a ferromagnet with a coordination number $z$, Eq. (31) then reduces to

$$
m=\left\langle\mu_{i}\right\rangle=\left.[\cosh (J \nabla)+m \sinh (J \nabla)]^{z} \tanh (\beta x)\right|_{x=0} .
$$


The transition temperature $T_{\mathrm{c}}$ can be obtained by linearizing (32); by expanding the right-hand side of (32) and taking only the linear term of $m$, one obtains

$$
\left.z \sinh (J \nabla) \cosh ^{z-1}(J \nabla) \tanh \left(\beta_{\mathrm{c}} x\right)\right|_{x=0}=1,
$$

where $\beta_{\mathrm{c}}=1 / k_{\mathrm{B}} T_{\mathrm{c}}$. In particular, when $z=6$ (or a simple cubic lattice), Eq. (33) reduces to

$$
\tanh \left(6 J \beta_{\mathrm{c}}\right)+4 \tanh \left(4 J \beta_{\mathrm{c}}\right)+5 \tanh \left(2 J \beta_{\mathrm{c}}\right)=\frac{16}{3},
$$

which is nothing but the result obtained by Zernike by means of another approach [7]. The transition temperature $T_{\mathrm{c}}$ is then given by

$$
\frac{k_{\mathrm{B}} T_{\mathrm{c}}}{J}=5.073 \quad \text { for } z=6 \text {, }
$$

which is superior to the MFA result

$$
\frac{k_{\mathrm{B}} T_{\mathrm{c}}}{J}=z \text {. }
$$

We are now in a position to clarify the background why the simple decoupling approximation (30) improves the standard MFA (14). For $H=0.0$, Eq. (31) can be also rewritten as follows:

$$
\left\langle\mu_{i}\right\rangle=\left.\prod_{j}\left[\frac{1}{2}\left(1+\left\langle\mu_{j}\right\rangle\right) \mathrm{e}^{J_{i j} \nabla}+\frac{1}{2}\left(1-\left\langle\mu_{j}\right\rangle\right) \mathrm{e}^{-J_{i j} \nabla}\right] \tanh (\beta x)\right|_{x=0} .
$$

Here, the factors $(1 / 2)\left(1+\left\langle\mu_{j}\right\rangle\right)$ and $(1 / 2)\left(1-\left\langle\mu_{j}\right\rangle\right)$ mean the probabilities of a neighboring spin $\mu_{j}$ being up and down. Then, exponential operators $\exp \left(J_{i j} \nabla\right)$ and $\exp \left(-J_{i j} \nabla\right)$ express in a sense $\exp \left(J_{i j} \nabla\right)$ for $\mu_{i}=+1$ and $\exp \left(-J_{i j} \nabla\right)$ for $\mu_{i}=-1$, respectively. On the other hand, the standard MFA consists of assuming that the field at the site $i$ is $\left\langle E_{i}\right\rangle=\sum_{j} J_{i j}\left\langle\mu_{j}\right\rangle$ independent of the orientation of $\mu_{i}$. This is clearly an approximation, for if $\mu_{i}$ is up, its neighbors $\mu_{j}$ will have more than average production for being up, a fluctuation effect that is neglected in the MFA. Thus, the partial correlation is included automatically in the simple framework through the usage of (20).

When one takes long-range interactions and the number of near neighbors goes to infinite, it is known that the MFA becomes to be exact. Within the present framework, let us here show this fact. For this aim, we take the exchange interaction $J_{i j}$ in (31) as

$$
J_{i j}=\frac{j}{N} \quad(j=\text { a finite constant }),
$$

where $N$ is the total number of lattice points. Then, Eq. (31) reduces to

$$
m=\left\langle\mu_{i}\right\rangle=\left.\left[\cosh \left(\frac{j}{N} \nabla\right)+m \sinh \left(\frac{j}{N} \nabla\right)\right]^{N-1} \tanh (\beta x+h)\right|_{x=0} .
$$

For a large value of $N, \cosh (j \nabla / N)$ and $\sinh (j \nabla / N)$ can be approximated as

$$
\cosh \left(\frac{j}{N} \nabla\right) \approx 1 \text { and } \sinh \left(\frac{j}{N} \nabla\right) \approx \frac{j}{N} \nabla
$$

so that (39) reduces to

$$
m=\left.\left[1+m \frac{j}{N} \nabla\right]^{N-1} \tanh (\beta x+h)\right|_{x=0}
$$


For $N \rightarrow \infty$, Eq. (40) is given by

$$
m=\left.\mathrm{e}^{N[m(j / N) \nabla]} \tanh (\beta x+h)\right|_{x=0}=\tanh (h+\beta m j) .
$$

Thus, the MFA result can be derived from the present framework, when $N \rightarrow \infty$. Finally, it will be fair to note some historical developments related to the framework of this part in the spin-1/2 Ising model. In order to treat the multispin correlation functions which appear for reducing the transcendental function to a polynomial form (or (15)), the decoupling approximation (30) was also introduced by Matsudaira [3]. He called it the first-order approximation. As noted above, the same decoupling approximation has been introduced into the present framework. It has been called the effective-field theory with correlations (EFT). The differential operator technique can be also rewritten in terms of the functional integration method [8]. Within the same framework as that of the EFT (or (30)), the method has been used by Lódź group [9]. Later, the same method as that of Matsudaira was proposed by Boccara [10], who was apparently unaware of these earlier works, and it has subsequently been used extensively by him and group of researchers in Morocco as the finite cluster approximation [11]. Clearly, as far as the physics is concerned, it is immaterial whether one uses Matsudaira's first-order approximation, the EFT, the functional integration methods or the finite cluster approximation. All of them correspond to the Zernike approximation. Ilowever, in these methods, the differential operator technique has generally been more favored, because of the relative easiness of the formulation of other thermodynamic properties and the extension to higher spin problems as well as disordered spin systems.

\subsection{Correlaled effective-field (or Bethe-Peierls) approximation}

In Sec. 4.1 we have introduced a simple decoupling method (30) for treating the multispin correlation functions. In this part, we shall discuss how the formulation of Sec. 4.1 can be improved to a better one (or from Zernike to Bethe-Peierls approximation).

Let us now assume that the nearest-neighbor Ising variable $\mu_{i+\delta}$ can be related to the central spin $\mu_{i}$ via

$$
\mu_{i+\delta}=\left\langle\mu_{i+\delta}\right\rangle+\lambda\left(\mu_{i}-\left\langle\mu_{i}\right\rangle\right)
$$

where $\lambda$ is a temperature dependent parameter. It is basically a measure of the short-range order, or pair correlation parameter.

When Eq. (42) is substituted into the IIamiltonian (1) with $H=0.0$, it is given by, for a system with nearest-neighbor interaction $J$,

$$
H=-\sum_{i} H_{i}^{\mathrm{eff}} \mu_{i}+\text { constant term }
$$

with

$$
H_{i}^{\mathrm{eff}}=J \sum_{j}\left\langle\mu_{j}\right\rangle-\lambda J z\left\langle\mu_{i}\right\rangle=H_{i}^{\mathrm{mol}}-R\left\langle\mu_{i}\right\rangle,
$$

where $R=\lambda J z$ is the parameter which has to be determined at the end of calculation in some way. This transformation to the one-body Hamiltonian (44) has 
been introduced by Lines [12] and then the effective-field $H_{i}^{\text {eff }}$ is modified by a term $-R\left\langle\mu_{i}\right\rangle$ from the standard mean-field $H_{i}^{\text {mol }}$.

This revision of the effective field is closely related to the fundamental concept introduced by Onsager for dielectrics [13]. He has discussed that the orienting part of the local field on a given dipole (or the cavity field) should not include the contribution arising from the part of the polarization of dipoles in its vicinity which comes from its instantaneous orientation (or the reaction field). Namely, the cavity field is then obtained from the total mean field by subtracting the mean reaction field

$$
E_{i}^{\text {cavity }}=\left\langle E_{i}\right\rangle-R\left\langle\mu_{i}\right\rangle .
$$

Thus, the effective field (44) is nothing but the cavity field (45) and the term $R\left\langle\mu_{i}\right\rangle$ corresponds to the reaction field. In the Lines method, the parameter $\lambda$ (or $R$ ) has been determined at the end of the calculation by imposing consistency of the theory wilh the sum rule for the susceptibility. However, the method gives an accuracy essentially equivalent to that of the spherical model [14], and unfortunately the sum rule is valid often only in the paramagnetic phase and in the absence of strong fields. Moreover, when the method is applied to the two-dimensional ferromagnetic Ising lattice, it generally predicts $T_{\mathrm{c}}=0$.

In the differential operator technique, on the other hand, the concept (44) has been used for evaluating the multispin correlation functions [15]. This is sharply in contrast to the above approach. Then, the parameter $\lambda$ has been determined self-consistently using the correlation function (23).

Substituting (42) into (23) with $\left\{f_{i}\right\}=1$ and taking the nearest-neighbor interactions, one obtains, on assuming that $m=\left\langle\mu_{i}\right\rangle=\left\langle\mu_{i+\delta}\right\rangle$ and $H=0.0$,

$$
\begin{aligned}
m & =\left.\left\langle\left\{P(m ; J \nabla)+\lambda\left[\cosh (J \nabla)+\mu_{i} \sinh (J \nabla)\right]\right\}^{z}\right\rangle \tanh (\beta x)\right|_{x=0} \\
& =\left.\left\langle\left[P(m ; J \nabla)+\lambda \mathrm{e}^{\mu_{i} J \nabla}\right]^{z}\right\rangle \tanh (\beta x)\right|_{x=0} \\
& =\left.\sum_{\nu=0}^{z} \frac{z !}{\nu !(z-\nu) !} \lambda^{\nu}[P(m ; J \nabla)]^{z-\nu}\left(\mathrm{e}^{\mu_{i} \nu J \nabla}\right) \tanh (\beta x)\right|_{x=0} \\
& =\left.\sum_{\nu=0}^{z} \frac{z !}{\nu !(z-\nu) !} \lambda^{\nu}[P(m ; J \nabla)]^{z-\nu}[\cosh (\nu J \nabla)+m \sinh (\nu J \nabla)] \tanh (\beta x)\right|_{x=0}
\end{aligned}
$$

with

$$
P(m ; J \nabla)=(1-\lambda)[\cosh (J \nabla)+m \sinh (J \nabla)] .
$$

Ilere, when $\lambda=0.0$, Eq. (46) reduces to (32).

For the evaluation of $\lambda$, on the other hand, let us use the two spin correlation function which is given by, on putting $\left\{f_{i}\right\}=\mu_{i+\delta}$ into (23),

$$
\begin{aligned}
& \left\langle\mu_{i+\delta} \mu_{i}\right\rangle=\left\langle\left[\sinh (J \nabla)+\mu_{i+\delta} \cosh (J \nabla)\right]\right. \\
& \left.\quad \times \prod_{\delta^{\prime}(\neq \delta)}\left[\cosh (J \nabla)+\mu_{i+\delta^{\prime}} \sinh (J \nabla)\right]\right\rangle\left.\tanh (\beta x)\right|_{x=0} .
\end{aligned}
$$


Substituting (42) into (48), one obtains

$$
\begin{aligned}
& \left\langle\mu_{i+\delta} \mu_{i}\right\rangle=m^{2}+\lambda\left(1-m^{2}\right)=\left\langle\left[\bar{P}(m ; J \nabla)+\lambda \mu_{i} \mathrm{e}^{\mu_{i} J \nabla}\right]\right. \\
& \left.\times\left[P(m ; J \nabla)+\lambda \mathrm{e}^{\mu_{i} J \nabla}\right]^{z-1}\right\rangle\left.\tanh (\beta x)\right|_{x=0} \\
& =\sum_{\nu=0}^{z-1} \frac{(z-1) !}{\nu !(z-1-\nu) !} \lambda^{\nu} P(m ; J \nabla)[P(m ; J \nabla)]^{z-1-\nu} \\
& \times\left.[\cosh (\nu J \nabla)+m \sinh (\nu J \nabla)] \tanh (\beta x)\right|_{x=0}+\sum_{\nu=0}^{z-1} \frac{(z-1) !}{\nu !(z-1-\nu) !} \lambda^{\nu+1} \\
& \times\left.[P(m ; J \nabla)]^{z-1-\nu}[m \cosh ((\nu+1) J \nabla)+\sinh ((\nu+1) J \nabla)] \tanh (\beta x)\right|_{x=0}
\end{aligned}
$$

with

$$
\bar{P}(m ; J \nabla)=(1-\lambda)[m \cosh (J \nabla)+\sinh (J \nabla)] .
$$

Thus, the magnetization $m$ and correlated parameter $\lambda$ of the Ising ferromagnet with a coordination number $z$ can be evaluated from the coupled equations (46) and (49).

For example, when $z=4$ (or square lattice), they reduce to

$$
m=4\left(K_{1}+3 K_{2} \lambda^{2}-2 K_{2} \lambda^{3}\right) m+4 K_{2}\left(1-3 \lambda^{2}+2 \lambda^{3}\right) m^{3}
$$

and

$$
\begin{aligned}
m^{2}+ & \lambda\left(1-m^{2}\right)=K_{1}\left(1+3 \lambda^{2}\right)+K_{2} \lambda^{2}\left(3+\lambda^{2}\right) \\
& +m^{2}\left[3 K_{1}\left(1-\lambda^{2}\right)+K_{2}\left(3+3 \lambda^{2}-8 \lambda^{3}+2 \lambda^{4}\right)\right] \\
& +m^{4}\left[1-6 \lambda^{2}+8 \lambda^{3}-3 \lambda^{4}\right],
\end{aligned}
$$

where coefficients $K_{1}$ and $K_{2}$ are given by

$$
\begin{aligned}
& K_{1}+\frac{1}{8}[\tanh (4 \beta J)+2 \tanh (2 \beta J)], \\
& K_{2}+\frac{1}{8}[\tanh (4 \beta J)-2 \tanh (2 \beta J)] .
\end{aligned}
$$

Thus, the transition temperature $T_{\mathrm{c}}$ can be determined from the coupled equations

$$
\begin{aligned}
& 1=4\left(K_{1}+3 K_{2} \lambda^{2}-2 K_{2} \lambda^{3}\right), \\
& \lambda=K_{1}+3 \lambda^{2}\left(K_{1}+K_{2}\right)+K_{2} \lambda^{4},
\end{aligned}
$$

which can be solved analytically and gives

$$
\frac{k_{\mathrm{B}} T}{J}=\frac{2}{\ln 2} \quad \text { and } \quad \lambda\left(T=T_{\mathrm{c}}\right)=\frac{1}{3} .
$$

The temperature dependence of $\lambda$ for the ferromagnetic square lattice with nearest-neighbor interaction $J$ is depicted in Fig. 1 by solving the coupled equations (50) and (51) numerically.

In general, the transition temperature $T_{\mathrm{c}}$ and the correlated parameter $\lambda$ at $T=T_{\mathrm{c}}$ are given by, within the present formulation (or (46) and (49)),

$$
\frac{k_{\mathrm{B}} T_{\mathrm{c}}}{J}=\frac{2}{\ln [z /(z-2)]}
$$




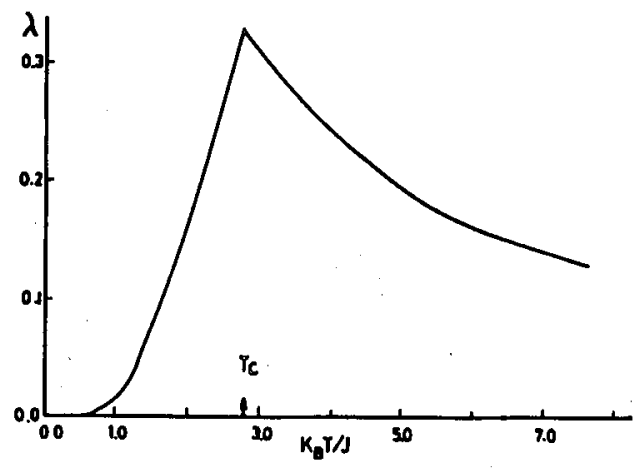

Fig. 1. The temperature dependence of $\lambda$ for the ferromagnetic square lattice.

Values of $k_{\mathrm{B}} T_{\mathrm{c}} / J$.

\section{TABLE I}

\begin{tabular}{r|r|c|c|l}
\hline \hline$z$ & MFA & EFT (Zernike) & Bethe-Peierls & Exact \\
\hline 2 & 2.0 & 0.0 & 0.0 & 0.0 \\
3 & 3.0 & 2.104 & 1.821 & 1.519 \\
4 & 4.0 & 3.090 & 2.885 & 2.269 \\
6 & 6.0 & 5.073 & 4.933 & 4.511 \\
8 & 8.0 & 7.061 & 6.952 & 6.353 \\
12 & 12.0 & 11.045 & 10.970 & 9.795
\end{tabular}

and

$$
\lambda\left(T=T_{c}\right)=\frac{1}{z-1} .
$$

The result of $T_{\mathrm{c}}$ is equivalent to that of Bethe-Peierls approximation, although the philosophy on which these two theories are based is different to each other. For comparison, the values of $T_{\mathrm{c}}$ obtained from Secs. 4.1 and 4.2 as well as the MFA are collected in Table $I$ and the exact or high-temperature series expansion results [16] are also listed.

\subsection{Effective-field renormalization group method}

In Secs. 4.1 and 4.2 we have discussed how the spin correlations can be decoupled for transforming the transcendental function into a polynomial form. Then, the results applicable to general lattice coordination numbers are obtained. However, the fault of these approaches is that the results depend on the coordination number, but not on the dimensionality. A value of $z=4$, for example, may equally be a square lattice or a diamond lattice. In order to take account of the lattice dimensionality as well as the coordination number, one has to treat 
the multispin correlation functions in forms depending on these qualities. Such a formulation can be made by going to Matsudaira's higher order decoupling approximation [3] better than the simple decoupling approximation (30). Then, the formulation cannot be described in a general form but it must be made separately in a way of depending on the lattice structure. Another way of incorporating these properties is to express the thermal average of the transcendental function as an average over a finite polynomial of spin operators in an $n$-site cluster $(n>1)$ $[4,17]$.

In this part, let us discuss how traditional (effective-field) procedures of obtaining equations of state can be converted into a modern tool for constructing a regular renormalization-group mapping according to the Wilson ideas. Due to its connection with standard mean-field procedure, the denomination of mean-field renormalization group has been used in the literature [18]. It has been successfully used to provide qualitative and quantitative insights into the critical behavior of spin systems. On the other hand, the effective-field renormalization-group scheme can be, via the differential operator technique, formulated by treating the effects of the surrounding spins of each of the clusters in a way of constructing the effective-field equations of states on the basis of the Ising spin identities.

The principle of the phenomenological renormalization group is based on the comparison of two clusters of different sizes $N, N^{\prime}\left(N^{\prime}<N\right)$, each of them simulating the infinite system. For the two clusters, one calculates an approximate equation of state for the magnetization per site, namely $m_{N}$ and $m_{N^{\prime}}$. In the mean-field renormalization group, this is done within the traditional mean-field scheme, in which the effects of the surrounding spins in each cluster is replaced by very small symmetric breaking fields $b$ and $b^{\prime}$, acting on the boundary sites of each of the clusters with $N$ and $N^{\prime}$ interacting spins, respectively. By imposing that both magnetizations of the clusters and respective symmetric breaking fields are scaled in the same way, one gets

$$
\left.\frac{\partial m_{N}(K, b)}{\partial b}\right|_{b=0}=\left.\frac{\partial m_{N^{\prime}}\left(K^{\prime} ; b^{\prime}\right)}{\partial b^{\prime}}\right|_{b^{\prime}=0},
$$

which is independent of the scaling factor. This relation gives a recursion relation between the coupling constants $K$ and $K^{\prime}$ in the systems. From the relation $K^{\prime}=K^{\prime}(K)$, the critical coupling $K_{\mathrm{c}}$ can be extracted by solving the fixed point equation $K^{*}=K^{\prime}\left(K^{*}\right)$ invariant under a change of scale. Furthermore, the critical exponent $\nu$ of the correlation length $\xi$ defined by

$$
\xi \propto\left|T-T_{\mathrm{c}}\right|^{-\nu}
$$

can be also obtained by linearizing the recursion relation in the neighborhood of the fixed point $K^{*}$ :

$$
\left(\frac{\partial K^{\prime}}{\partial K}\right)_{K=K^{\prime}}=l^{1 / \nu}
$$

where $l=\left(N / N^{\prime}\right)^{1 / d}$ is the scaling factor and $d$ is the dimensionality of the system.

Let us illustrate now the general arguments of the phenomenological renormalization group by taking the simplest choice, namely, clusters of one $\left(N^{\prime}=1\right)$ and two $(N=2)$ spins. In the one-spin cluster the spin $\mu_{1}$ interacts with $z_{1}$ 
nearest-neighbor sites via the coupling constants $K_{i j}^{\prime}$. In the two-spin cluster, on the other hand, the spins $\mu_{1}$ and $\mu_{2}$ interact directly via the coupling $K_{12}$ and both $\mu_{1}$ and $\mu_{2}$ spins interact with their neighbor sites also via the coupling constants $K_{1 i}$ and $K_{2 j}^{\prime}$. Using the same procedures as those of Sec. 2, the averaged magnetizations $m_{N^{\prime}}$ and $m_{N}$ associated to the $N^{\prime}=1$ and $N=2$ clusters are given by

$$
m_{N^{\prime}}=\left\langle\mu_{1}\right\rangle=\left\langle\tanh \left(\sum_{j} K_{1 j}^{\prime} \mu_{j}^{\prime}\right)\right\rangle
$$

and

$$
m_{N}=\left\langle\frac{1}{2}\left(\mu_{1}+\mu_{2}\right)\right\rangle=\left\langle\frac{\sinh (u+v)}{\cosh (u+v)+\exp \left(-2 K_{12}\right) \cosh (u-v)}\right\rangle,
$$

where $u=\sum_{j} K_{1 j} \mu_{j}$ and $v=\sum_{j^{\prime}} K_{2 j^{\prime}} \mu_{j^{\prime}}$.

Using the differential operator technique and noticing that the sites 1 and 2 of the two-spin cluster may include a set of common-neighbor sites, the set of Eqs. (61) and (62) can be written in the following forms:

$$
m_{N^{\prime}}=\left.\left\langle\prod_{j} \exp \left(K_{1 j}^{\prime} \mu_{j}^{\prime} \nabla_{x}\right)\right\rangle f(x)\right|_{x=0}
$$

and

$$
\begin{aligned}
m_{N} & =\left\langle\prod_{j}^{\prime} \exp \left(K_{1 j} \mu_{j} \nabla\right) \prod_{j^{\prime}}^{\prime} \exp \left(K_{2 j^{\prime}} \mu_{j}, \nabla_{y}\right)\right. \\
& \left.\times \prod_{k}^{\prime} \exp \left[\mu_{k}\left(K_{1 k} \nabla_{x}+K_{2 k} \nabla_{y}\right)\right]\right\rangle\left. f(x, y)\right|_{x=0, y=0}
\end{aligned}
$$

where $\nabla_{\mu}=\partial / \partial \mu(\mu=x$ or $y)$ are the differential operators and the functions $f(x)$ and $f(x, y)$ are defined by

$$
f(x)=\tanh x
$$

and

$$
f(x, y)=\frac{\sinh (x+y)}{\cosh (x+y)+\exp \left(-2 K_{12}\right) \cosh (x-y)} .
$$

Here, the products $\Pi^{\prime}$ over $j$ and $j^{\prime}$ in (64) are respectively the isolated nearest-neighbor spins of siles 1 and 2 , while the product $\Pi^{\prime}$ over $k$ is restricted to the sites which are simultaneously nearest neighbors of both $\mu_{1}$ and $\mu_{2}$ spins. Furthermore, the exponential operators in (63) and (64) can be rewritten into the product forms of $\mu_{j}$ by the use of $(20)$.

As discussed in Sec. 4.1, we introduce here the decoupling approximation (30) into the exact relations (63) and (64). Basing on the approximation (30) and replacing each boundary average $\left\langle\mu_{j^{\prime}}\right\rangle$ (or $\left.\left\langle\mu_{j}\right\rangle\right)$ in their right-hand sides with the symmetry breaking mean-field parameters $b_{j}^{\prime}$ (or $b_{j}$ ), the critical behavior of the system can be obtained by expanding the right-hand side of them and taking only first-order terms in these parameters

$$
m_{N^{\prime}}\left(K^{\prime}, b^{\prime}\right)=A_{N^{\prime}}^{(z)}\left(K^{\prime}\right) b^{\prime}+\mathrm{O}\left(b^{3}\right)
$$


and

$$
m_{N}(K, b)=A_{N}^{(z)}(K) b+\mathrm{O}\left(b^{3}\right),
$$

where the coefficients $A_{N^{\prime}}^{(z)}\left(K^{\prime}\right)$ and $A_{N}^{(z)}(K)$ for the $N^{\prime}=1$ and $N=2$ clusters are given, on assuming only the nearest-neighbor interactions $\left(K^{\prime}\right.$ and $\left.K\right)$, by

$$
A_{N^{\prime}}^{(z)}\left(K^{\prime}\right)=\left.z_{1} \cosh ^{z-1}\left(K \nabla_{x}\right) f(x)\right|_{x=0}
$$

and

$$
\begin{aligned}
& A_{N}^{(z)}(K)=\left\{2 z^{\prime} \sinh \left(K \nabla_{x}\right) \cosh ^{z^{\prime}-1}\left(K \nabla_{x}\right) \cosh ^{z^{\prime}}\left(K \nabla_{y}\right) \cosh ^{z^{\prime \prime}}\left[K\left(\nabla_{x}+\nabla_{y}\right)\right]\right. \\
& +z^{\prime \prime} \sinh \left[K\left(\nabla_{x}+\nabla_{y}\right)\right] \cosh ^{z^{\prime}}\left(K \nabla_{x}\right) \cosh ^{z^{\prime}}\left(K \nabla_{y}\right) \\
& \left.\times \cosh ^{z^{\prime \prime}-1}\left[K\left(\nabla_{x}+\nabla_{y}\right)\right]\right\}\left.f(x, y)\right|_{x=0, y=0^{\circ}}
\end{aligned}
$$

Here, $z^{\prime}$ denotes the number of sites which are nearest neighbors of $\mu_{1}$ (or $\mu_{2}$ ) but not neighboring to $\mu_{2}$ (or $\mu_{1}$ ), and $z^{\prime \prime}$ represents the number of sites that are simultaneously nearest neighbors of both $\mu_{1}$ and $\mu_{2}$. Thus, $z_{2}=2 z^{\prime}+z^{\prime \prime}$ is the total number of nearest-neighbour sites of the two-spin cluster. Hence, the coefficient $A_{N}^{(z)}(K)$ incorporates the detail of the geometry of the lattice beyond its coordination number $z_{1}$, through $z^{\prime}$ and $z^{\prime \prime}$.

Combining (69) and (70) with the scaling assumption, one gets from (58)

$$
A_{N^{\prime}}^{(z)}\left(K^{\prime \prime}\right)=A_{N}^{(z)}(K) \text {, }
$$

which is the recursion relation between the coupling constants $K$ and $K^{\prime}$ for the two rescaled systems $N^{\prime}=1$ and $N=2$. The reduced critical interaction $K_{\mathrm{c}}$ is the non-trivial fixed point $K^{\prime}=K=K^{*}=K_{\mathrm{c}}$ solution of (71) and the critical exponent $\nu$ for the correlation length can be obtained from (60), noting that

$$
\left(\frac{\partial K^{\prime}}{\partial K}\right)_{K=K}=\frac{\left(\frac{\partial A_{N}^{(x)}(K)}{\partial K}\right)_{K=K}}{\left(\frac{\partial A_{N N^{\prime}\left(K^{\prime}\right)}^{(x)}}{\partial K^{\prime}}\right)_{K=K^{*}}} .
$$

These approaches can be also extended to higher-order approximate recursion relations by considering clusters larger than $N=2$. The numerical results obtained from such effective-field renormalization group methods are given in $\mathrm{Ta}$ ble II and compared with those of the exact calculations or high-temperature series expansion methods as well as the mean-field renormalization group method [19].

\section{Thermodynamic properties}

In the previous sections, some magnetic properties ( $T_{\mathrm{c}}$ and magnetization) of a spin-1/2 Ising system have been discussed. Here, let us study how other thermodynamic properties of a spin-1/2 Ising system can be formulated on the basis of the differential operator technique. 
TABLE II

(a) Values of the reduced critical temperature $K_{c}^{-1}$ for several lattice structures [19] and the mean-field renormalization group (MFRG) (the numbers in brackets) [18], when $N^{\prime}=1$ and $N=2$. (b) Results for the reduced critical temperature $K_{\mathrm{c}}^{-1}$ and critical exponent $\nu$. for the simple quadratic and three-dimensional cubic Ising models [19], when higher-order approximate recursion relations are used.

\begin{tabular}{c|c|c|c}
\hline \hline \multicolumn{4}{|c}{ (a) } \\
\hline & Honeycomb & Kagome & Quadratic \\
\hline$\left(d, z_{1}, z_{2}\right)$ & $(2,3,4)$ & $(2,4,5)$ & $(2,4,6)$ \\
EFRG(MFRG) & $1.588(1.820)$ & $2.083(2.882)$ & $2.794(2.882)$ \\
\hline
\end{tabular}

(b)

\begin{tabular}{c|c|c|c|c}
\hline \multirow{2}{*}{$N / N^{\prime}$} & \multicolumn{2}{|c|}{ Simple quadratic } & \multicolumn{2}{c}{ Simple cubic } \\
\cline { 2 - 5 } & $K_{\mathrm{c}}^{-1}$ & $\nu$ & $K_{\mathrm{c}}^{-1}$ & $\nu$ \\
\hline $2 / 1$ & $2.794(2.882)$ & $1.393(1.667)$ & $4.852(4.926)$ & $1.369(1.538)$ \\
$4 / 1$ & $2.697(2.770)$ & $1.166(1.449)$ & $4.829(4.902)$ & $1.238(1.389)$ \\
$4 / 2$ & $2.640(2.703)$ & $1.006(1.282)$ & $4.806(4.878)$ & $1.139(1.266)$ \\
Exact & 2.269 & 1.00 & 4.511 & 0.629
\end{tabular}

\subsection{Initial susceptibility}

The initial susceptibility $\chi$ is defined by

$$
\chi(T)=\left.\frac{\partial m(T, H)}{\partial H}\right|_{H=0} .
$$

For the spin-1/2 Ising model, the magnetization $m(T, H)$ is given by (23), on putting $\left\{f_{1}\right\}=1$ into it. Accordingly, the initial susceptibility (73) is given by

$$
\begin{aligned}
& \chi(T)=\left.\left\{\frac{\partial}{\partial H}\left\langle\prod_{j}\left[\cosh \left(J_{i j} \nabla\right)+\mu_{j} \sinh \left(J_{i j} \nabla\right)\right]\right\rangle\right\}_{H=0} \tanh (\beta x)\right|_{x=0} \\
& +\left.\beta\left\langle\prod_{j}\left[\cosh \left(J_{i j} \nabla\right)+\mu_{j} \sinh \left(J_{i j} \nabla\right)\right]\right\rangle_{0} \operatorname{sech}^{2}(\beta x)\right|_{x=0}
\end{aligned}
$$

where $\langle\cdots\rangle_{H}$ and $\langle\cdots\rangle_{0}$ express respectively the field- and zero-field dependencies of the canonical average. This is also exact and valid for any lattice.

For the spin-1/2 linear chain with nearest-neighbor interactions, Eq. (74) can be calculated exactly by the use of the spin correlation function $\left\langle\mu_{i-1} \mu_{i+1}\right\rangle_{0}$ as follows [5]:

$$
\chi=\frac{1}{2 k_{\mathrm{B}} T} \frac{\left[1+\left\langle\mu_{i-1} \mu_{i+1}\right\rangle_{0}\right] \operatorname{sech}^{2}(2 \beta J)+\left[1-\left\langle\mu_{i+1} \mu_{i-1}\right\rangle_{0}\right]}{1-\tanh (2 \beta J)} .
$$


From Eq. (29), $\left\langle\mu_{i-1} \mu_{i+1}\right\rangle_{0}$ is given by $\tanh ^{2}(\beta J)$, so that (75) reduces to

$$
\chi(T)=\frac{1}{k_{\mathrm{B}} T} \exp \left(2 J / k_{\mathrm{B}} T\right),
$$

which is the exact result of the spin-1/2 Ising chain.

In a real system with a higher dimensional lattice where the number of neighbors is $z>2$, one cannot calculate (74) exactly and hence some approximation must be introduced for the evaluation. For example, when the decoupling approximation (30) is introduced into the right-hand side of (74), the initial susceptibility of the spin-1/2 Ising ferromagnet with nearest-neighbor interactions can be expressed as

with

$$
\chi(T)=\frac{\Delta}{k_{\mathrm{B}} T(1-\Gamma)}
$$

$$
\begin{aligned}
& \Gamma=\left.z \sinh (J \nabla)[\cosh (J \nabla)+m \sinh (J \nabla)]^{z-1} \tanh (\beta x)\right|_{x=0}, \\
& \Delta=\left.[\cosh (J \nabla)+m \sinh (J \nabla)]^{z} \operatorname{sech}^{2}(\beta x)\right|_{x=0},
\end{aligned}
$$

where the magnetization $m$ can be obtained from (32). At $T=T_{\mathrm{c}}, m=0$, so that the relation $\Gamma=1$ in the denominator of (77) reduces to (27). From it, the transition temperature can be determined. Thus, the initial susceptibility diverges at $T=T_{\mathrm{c}}$. The paramagnetic susceptibility valid for $T>T_{\mathrm{c}}$ is then obtained by substituting $m=0$ into (78) and (79).

\subsection{Internal energy and specific heat}

For $H=0.0$, the internal energy $U$ of the spin-1/2 Ising model is given by

$$
\frac{U}{N}=-\frac{1}{2}\left\langle E_{i} \mu_{i}\right\rangle
$$

where $N$ is the total number of magnetic atoms and $E_{i}=\sum_{j} J_{i j} \mu_{j}$. Substituting $\left\{f_{i}\right\}=E_{i}$ into (23), the internal energy can be written as

$$
\begin{aligned}
\frac{2 U}{N} & =-\left.\left\langle E_{i} \mathrm{e}^{E_{i} \nabla}\right\rangle \tanh (\beta x)\right|_{x=0} \\
& =-\left.\left[\frac{\partial}{\partial y}\left\langle\exp \left(E_{i} y\right)\right\rangle\right]_{y=\nabla} \tanh (\beta x)\right|_{x=0} .
\end{aligned}
$$

This is also exact. Then, the specific heat, defined as

$$
C(T)=\frac{\partial U}{\partial T},
$$

can be also evaluated by using (81).

For the spin-1/2 Ising chain, the internal energy (81) can be calculated exactly. It is given by

$$
\frac{2 U}{N}=-J \tanh (2 \beta J)\left[1+\left\langle\mu_{i-1} \mu_{i+1}\right\rangle\right]=-2 J \tanh (\beta J),
$$

from which the specific heat is

$$
C=\frac{J^{2} N}{k_{\mathrm{B}} T^{2}} \operatorname{sech}^{2}\left(J / k_{\mathrm{B}} T\right) \text {. }
$$


Thus, the exact thermodynamic properties of the spin- $1 / 2$ Ising chain can be derived from the differential operator scheme.

For real systems, on the other hand, the expression $\left\langle\exp \left(E_{i} y\right)\right\rangle$ in $(81)$ can be evaluated by the use of some approximation discussed in Sec. 4 . Within the framework of the decoupling approximation (30), the internal energy of the spin-1/2 Ising ferromagnet with nearest-neighbor interactions is given by

$$
\begin{aligned}
\frac{2 U}{N}= & -z J[\sinh (J \nabla)+m \cosh (J \nabla)] \\
& \times\left.[\cosh (J \nabla)+m \sinh (J \nabla)]^{z-1} \tanh (\beta x)\right|_{x=0},
\end{aligned}
$$

from which the specific heat can be also obtained. Here, notice that the decoupling (or Zernike) approximation predicts a magnetic specific heat starting from zero at $T=0 \mathrm{~K}$ and rising to a sharp maximum at $T=T_{\mathrm{c}}$. At this point it drops discontinuously to a non-zero value and then tails off at high temperatures, varying as $T^{-2}$ in the high-temperature limit. As is understood from (85) with $m=0.0$, the non-vanishing specific heat provides a distinct improvement over the traditional MFA with $C=0.0$ for $T>T_{\mathrm{c}}$, due to the finite short-range order above the Curie point $[4,20]$.

\section{Spin'one Ising models}

In the previous sections, the spin- $1 / 2$ Ising model has been formulated and discussed on the basis of the differential operator technique. On the other hand, spin-one Ising models are also encountered in different fields of physics and continue to be one of the most actively studied problems in statistical mechanics. In contrast to the spin-1/2 Ising model, they are of particular importance, because of the fundamental interest in the multicritical phenomena of physical systems, such as ${ }^{3} \mathrm{H}_{\mathrm{e}}{ }^{4} \mathrm{H}_{\mathrm{e}}$ mixtures, ternary alloys, metamagnets and multicomponent fluids. In particular, the spin-one Ising model with a crystal-field interaction is often called the Blume-Capel (BC) model [21] and the Blume-Emery-Griffiths (BEG) model [22] contains a biquadratic exchange interaction and single-ion anisotropy in addition to the bilinear exchange interaction. In this section, let us study how the two spin-one Ising models can be formulated by starting from the corresponding Ising spin identities and using the differential operator technique.

\subsection{Blume-Capel model}

The Hamiltonian of the Blume-Capel (BC) model is given by

$$
H=-J \sum_{i, j} S_{i}^{z} S_{j}^{z}-D \sum_{i}\left(S_{i}^{z}\right)^{2},
$$

where $S_{i}^{z}$ takes the values \pm 1 and 0 , and the first summation runs over all nearest-neighbor spins. $J(J>0)$ is the exchange interaction and $D-$ the crystal-field constant.

Using the treatment presented in Sec. 2, one can obtain the exact identity of this model as

$$
\left\langle\left\{f_{i}\right\} S_{i}^{z}\right\rangle=\left\langle\left\{f_{i}\right\} F\left(E_{i}\right)\right\rangle
$$


with

$$
E_{i}=J \sum_{j} S_{j}^{z},
$$

where the function $F(x)$ is defined by

$$
F(x)=\frac{2 \sinh (\beta x)}{2 \cosh (\beta x)+\exp (-\beta D)} .
$$

This identity corresponds to a special case of Eqs. (10) or (12). In fact, when $D \rightarrow \infty$, the function $F(x)$ reduces to $\tanh (\beta x)$ and hence (89) becomes equivalent to (10). In other words, when $D$ takes a large positive value, the $\mathrm{BC}$ model behaves like the standard spin-1/2 Ising model and in this case the $S_{i}^{z}=0$ state is not allowed energetically.

On the other hand, by comparing the values of the ground state energies for the BC model with $S_{i}^{z}= \pm 1$ or $S_{i}^{z}=0$, the possible ordered phases at $T=0 \mathrm{~K}$ are separated at the critical value $D_{\mathrm{c}}$ of $D$, namely

$$
\frac{D_{c}}{J}=-\frac{z}{2},
$$

where $z$ is the coordination number. That is to say, at the critical value, the ordered phases at $T=0 \mathrm{~K}$ may show the first-order transition: from the $S_{i}^{z}= \pm 1$ state to the $S_{i}^{z}=0$ state, when the value of $D$ decreases. Below the critical value, the $\mathrm{BC}$ model does not show any long-range order (or $m=\left\langle S_{i}^{z}\right\rangle=0.0$ ) at $T=0 \mathrm{~K}$. In relation to this behavior, the BC model may take a characteristic feature (or multicritical phenomenon), when the value of $D$ becomes negative. Thus, the BC model may exhibit an outstanding phenomenon completely different from the standard spin-1/2 Ising model.

Now, by introducing the differential operator technique (87) can be written as

$$
\left\langle\left\{f_{i}\right\} S_{i}^{z}\right\rangle=\left.\left\langle\left\{f_{i}\right\} \mathrm{e}^{E_{i} \nabla}\right\rangle F(x)\right|_{x=0} .
$$

For a system with $S=1 / 2$, we have used the identity (20), in order to treat the exponential operator $\exp \left(E_{i} \nabla\right)$. For this case, the $S_{i}^{z}$ takes three components, namely $S_{i}^{z}=+1,0,-1$, and hence we must use another identity given by

$$
\exp \left(a S_{i}^{z}\right)=\left(S_{i}^{z}\right)^{2} \cosh a+S_{i}^{z} \sinh a+1-\left(S_{i}^{z}\right)^{2}
$$

for $S=1$ [23]. Then, Eq. (91) can be written as

$$
\begin{aligned}
& \left\langle\left\{f_{i}\right\} S_{i}^{z}\right\rangle \\
& =\left.\left\langle\left\{f_{i}\right\} \prod_{j}\left[\left(S_{j}^{z}\right)^{2} \cosh (J \nabla)+S_{j}^{z} \sinh (J \nabla)+1-\left(S_{j}^{z}\right)^{2}\right]\right\rangle F(x)\right|_{x=0} .
\end{aligned}
$$

This is also exact and is valid for any lattice. In particular, the magnetization per site, $\left\langle S_{i}^{z}\right\rangle$, can be obtained by substituting $\left\{f_{i}\right\}=1$ into (93).

At this place, expanding the right-hand side of (93), multispin correlation functions appear. However, the formulations discussed in Sec. 4 can be also applied to this model with some modifications. The first step for treating them is to neglect the correlation between different sites, namely

$$
\left\langle S_{j}^{z}\left(S_{k}^{z}\right)^{2} \ldots S_{l}^{z}\right\rangle \approx\left\langle S_{j}^{z}\right\rangle\left\langle\left(S_{k}^{z}\right)^{2}\right\rangle \ldots\left\langle S_{l}^{z}\right\rangle,
$$


which corresponds to the Zernike approximation of the spin-1/2 Ising model. Introducing the approximation, the magnetization per site is given by

$$
\left\langle S_{i}^{z}\right\rangle=\left.\prod_{j}\left[\left\langle\left(S_{j}^{z}\right)^{2}\right\rangle \cosh (J \nabla)+\left\langle S_{j}^{z}\right\rangle \sinh (J \nabla)+1-\left\langle\left(S_{j}^{z}\right)^{2}\right\rangle\right] F(x)\right|_{x=0} .
$$

In order to evaluate $\left\langle S_{i}^{z}\right\rangle$, it is necessary here to calculate another order parameter $\left\langle\left(S_{i}^{z}\right)^{2}\right\rangle$. This situation normally appears in a system with $S>1 / 2$, when we use a method beyond the standard MFA. By the use of the same procedure as that of (91), one can also derive an exact identity

with

$$
\left\langle\left(S_{i}^{z}\right)^{2}\right\rangle=\left\langle G\left(E_{i}\right)\right\rangle=\left.\left\langle\mathrm{e}^{E_{i} \nabla}\right\rangle G(x)\right|_{x=0}
$$

$$
G(x)=\frac{2 \cosh (\beta x)}{2 \cosh (\beta x)+\exp (-\beta D)} .
$$

Assuming that

$$
m=\left\langle S_{i}^{z}\right\rangle \text { and } q=\left\langle\left(S_{i}^{z}\right)^{2}\right\rangle,
$$

we obtain, from (95) and (96),

$$
m=\left.[q \cosh (J \nabla)+m \sinh (J \nabla)+1-q]^{z} F(x)\right|_{x=0}
$$

and

$$
q=\left.[q \cosh (J \nabla)+m \sinh (J \nabla)+1-q]^{z} G(x)\right|_{x=0} .
$$

These equations are valid for any kind of lattice structure characterized by the coordination number $z$. In particular, for the linear chain $(z=2)$ one can easily prove from these equations that $m=0.0$ for all temperatures (or $T_{\mathrm{c}}=0$ ) [24]. IIere, for deriving Eqs. (99) and (100), we have used Eq. (94). The improvement may be also done, like in Secs. 4.2 and 4.3. For other thermodynamic properties, we can also formulate in the same way as that of spin-1/2 Ising model in Sec. 5 .

As noted first, the BC model may show a characteristic behavior (or a multicritical point), when the value of $D / J$ takes a negative number. That is to say, it is well known that the tricritical behavior may appear in the system. At the tricritical point, the system changes from the second-order phase transition to the first-order one, when the transition temperature is plotted as a function of $D$.

In order to discuss the tricritical behavior, the right-hand side of (99) and (100) must be expanded by assuming that $m$ is small. Then, we obtain in general an equation for $m$ of the form

$$
m=a m+b m^{3}+c m^{5}+\ldots
$$

The second-order phase transition line is determined by the condition

$$
a=1 \text { and } b<0 .
$$

IIere, the parameter $a$ is defined by

$$
a=\left.z \sinh (J \nabla)\left[q_{0} \cosh (J \nabla)+1-q_{0}\right]^{z-1} F(x)\right|_{x=0},
$$

where $q_{0}$ is the solution of

$$
q_{0}=\left.\left[q_{0} \cosh (J \nabla)+1-q_{0}\right]^{z} G(x)\right|_{x=0} .
$$


In the vicinity of the second-order phase transition line, the magnetization $m$-is given by

$$
m^{2}=\frac{1-a}{b} .
$$

The right-hand side must be positive. If it is not the case, the transition is of the first order, and hence the point at which

$$
a=1 \text { and } b=0
$$

is the tricritical point [25].

At this point, in order to obtain the expression for $b$, let us substitute

$q=q_{0}+q_{1} m^{2}$

into (100). The expression of $q_{1}$ is then given by

$$
q_{1}=\frac{f}{1-e}
$$

with

$$
\begin{aligned}
& e=\left.z\left[q_{0} \cosh (J \nabla)+1-q_{0}\right]^{z-1}[\cosh (J \nabla)-1] G(x)\right|_{x=0}, \\
& f=\left.\frac{z !}{2 !(z-2) !}\left[q_{0} \cosh (J \nabla)+1-q_{0}\right]^{z-2} \sinh ^{2}(J \nabla) G(x)\right|_{x=0} .
\end{aligned}
$$

Substituting (107) into (99), the expression of $b$ in (105) is given by

$$
\begin{aligned}
b= & \frac{z !}{(z-2) !} q_{1}\left[q_{0} \cosh (J \nabla)+1-q_{0}\right]^{z-2} \\
& \times\left.\sinh (J \nabla)[\cosh (J \nabla)-1] F(x)\right|_{x=0} \\
& +\left.\frac{z !}{3 !(z-3) !}\left[q_{0} \cosh (J \nabla)+1-q_{0}\right]^{z-3} \sinh ^{3}(J \nabla) F(x)\right|_{x=0} .
\end{aligned}
$$

In Fig. 2, the transition temperature of the BC model is schematically depicted as a function of $D / J$. In the figure, the solid and dashed lines denote respectively the second-order and first-order phase transition lines. The white circle represents the tricritical (or multicritical) point. In Table III, the results of the tricritical point obtained from the condition (106) as well as other methods are collected. These results indicate that the tricritical point $[24,25]$ of the BC model is universally found at

$$
\frac{D_{\mathrm{t}}}{z J}=-0.47
$$

\subsection{The Blume-Emery-Griffiths model}

The Hamiltonian of the Blume-Emery-Griffiths (BEG) model is given by

$$
H=-J \sum_{i, j} S_{i}^{z} S_{j}^{z}-J^{\prime} \sum_{i, j}\left(S_{i}^{z} S_{j}^{z}\right)^{2}-D \sum_{i}\left(S_{i}^{z}\right)^{2},
$$

where $J, J^{\prime}$, and $D$ are the bilinear, biquadratic and anisotropy parameters, respectively. Here, when $J^{\prime}=0.0$, Eq. (113) reduces to (86), namely the $\mathrm{BC}$ model. 


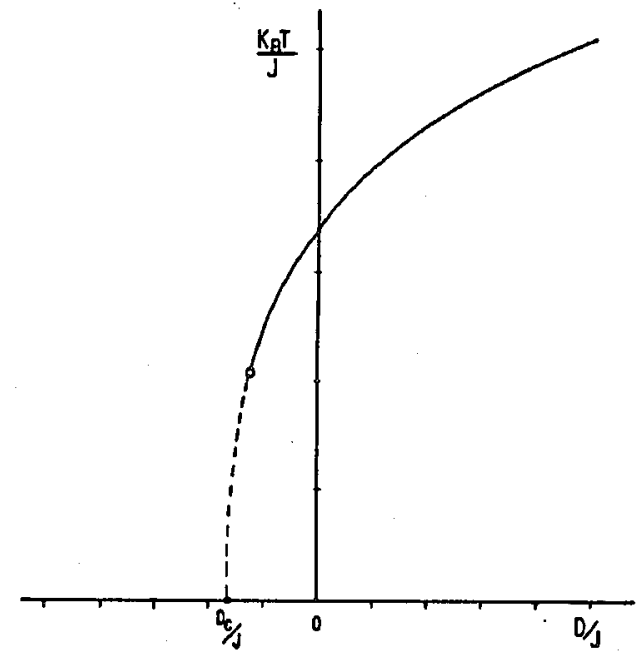

Fig. 2. Phase diagram of the spin-one $B C$ model. The white circle represents the tricritical point. The solid and dashed lines express respectively the second-order and first-order transition lines.

TABLE III

Values of tricritical points $\left(D_{\mathrm{t}} / z J, k_{\mathrm{B}} T_{\mathrm{t}} / z J\right)$ in the spin-one $\mathrm{BC}$ model.

\begin{tabular}{c|c|c|c|c}
\hline \hline$z$ & MFA & EFT & RG & SE \\
\hline 3 & - & $(-0.473,0.237)$ & - & - \\
4 & - & $(-0.472,0.253)$ & $(-0.501,0.232)$ & - \\
6 & $(-0.463,0.333)$ & $(-0.468,0.279)$ & $(-0.482,0.262)$ & $(-0.470,0.271)$
\end{tabular}

Accordingly, the frameworks discussed for the $\mathrm{BC}$ model can be straightforwardly extended to the BEG model, when the identities for the model are obtained.

For this aim, the exact identities of the BEG model were derived by Fittipaldi and Kaneyoshi [26], and Tucker [27] by the use of the differential operator technique. They are given by

$$
\begin{aligned}
& \left\langle\left\{f_{i}\right\} S_{i}^{z}\right\rangle=\left.\left\langle\left\{f_{i}\right\} \prod_{\delta} P_{i}\left(S_{i+\delta},\left(S_{i+\delta}\right)^{2} ; \nabla_{x}, \nabla_{y}\right)\right\rangle f(x, y)\right|_{x=0, y=0} \\
& \left\langle\left\{f_{i}\right\}\left(S_{i}^{z}\right)^{2}\right\rangle=\left.\left\langle\left\{f_{i}\right\} \prod_{\delta} P_{i}\left(S_{i+\delta},\left(S_{i+\delta}\right)^{2} ; \nabla_{x}, \nabla_{y}\right)\right\rangle g(x, y)\right|_{x=0, y=0}
\end{aligned}
$$

with

$$
P_{i}\left(S_{j}^{z},\left(S_{j}^{z}\right)^{2} ; \nabla_{x}, \nabla_{y}\right)=1+\alpha \rho S_{j}^{z}+(\gamma \rho-1)\left(S_{j}^{z}\right)^{2},
$$

in which we have introduced the notations

$$
\alpha=\sinh \left(K \nabla_{x}\right), \quad \gamma=\cosh \left(K \nabla_{x}\right), \quad \rho=\exp \left(K^{\prime} \nabla_{y}\right),
$$


where $K=\beta J, K^{\prime}=\beta J^{\prime}$ and $\nabla_{\mu}=\partial / \partial \mu(\mu=x, y)$ are the two differential operators. The functions $f(x, y)$ and $g(x, y)$ are defined by

and

$$
f(x, y)=\frac{2 \exp y \sinh x}{\exp (-\beta D)+2 \exp y \cosh x}
$$

$$
g(x, y)=\frac{2 \exp y \cosh x}{\exp (-\beta D)+2 \exp y \cosh x} .
$$

At this place, one should notice that the identity

$$
\exp \left(a S_{i}^{z}\right) \exp \left(a^{\prime}\left(S_{i}^{z}\right)^{2}\right)=1+S_{i}^{z} \exp a^{\prime} \sinh a+\left(S_{i}^{z}\right)^{2}\left[\exp a^{\prime} \cosh a-1\right]
$$

has been used for the derivation, instead of (92) in the BC model.

Owing to the similarity between the identities (91) and (96) of the BC model and the identities (114) and (115) of the BEG model, we can also introduce the approximate treatments of Sec. 6.1 into the identities (114) and (115). The simplest approximation is to use the decoupling approximation (94). Then, the expectation values defined by

$$
m_{i}=\left\langle S_{i}^{z}\right\rangle \text { and } q_{i}=\left\langle\left(S_{i}^{z}\right)^{2}\right\rangle
$$

are given by

$$
\begin{aligned}
& m_{i}=\left.\prod_{\delta}\left[1+\alpha \rho m_{i+\delta}+(\gamma \rho-1) q_{i+\delta}\right] f(x, y)\right|_{x=0, y=0} \\
& q_{i}=\left.\prod_{\delta}\left[1+\alpha \rho m_{i+\delta}+(\gamma \rho-1) q_{i+\delta}\right] g(x, y)\right|_{x=0, y=0}
\end{aligned}
$$

Now, let us define the parameters $d$ and $r$ as

$$
d=\frac{D}{J} \quad \text { and } \quad r=\frac{J^{\prime}}{J}
$$

where $J>0$. We assume at first that $m=m_{i}$ and $q=q_{i}$ in (122) and (123), like the BC model. This may be called the one-sublattice model. Then, from the ground state argument the $S_{i}^{z}=0(m=0, q=0)$ state and the $S_{i}^{z}= \pm 1(|m|=1$, $q=1)$ state at $T=0 \mathrm{~K}$ are separated by the condition

$$
d=-\frac{z}{2}(1+r)
$$

instead of (90). This relation is depicted in Fig. 3 as a dashed line. When $T_{\mathrm{c}}$ of the system with $r>-1$ is plotted as a function of $d$, the tricritical point can be also found on the curve at a negative value of $d$. On the other hand, when $r=-1.0$, the $T_{\mathrm{c}}$ curve reduces to zero at $d=0.0$ and hence the tricritical behavior as well as $T_{c}$ cannot be obtained in the negative region of $d$. In this way, the BEG model has an additional parameter $r$. Therefore, it can include richer phenomena than the BC model, especially when $r$ becomes smaller than $r=-1.0$. The situation is depicted in the figure, which is obtained from the magnetization process in the one-sublattice model [28].

A particular interest of recent researches is directed to the region of $r<-1.0$ in this model. The Monte Carlo simulation [29] has predicted that a new phase may occur in the region of $J+J^{\prime}<0.0$ and $D<0.0$, namely $r<-1.0$ and 


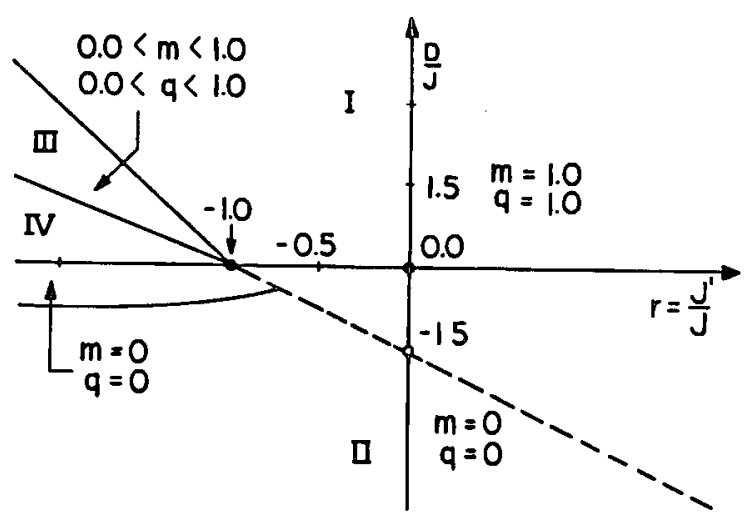

Fig. 3. The possible ground-state phase diagram expected from the saturation process for the BEG model on a honeycomb lattice.

$d<0.0$. In the phase the system does not show any long-range order (or $m=0.0$ ) in a usual sense and splits into two interpenetrating sublattices. That is to say, the result indicates that the new disordered phase consists of two interpenetrating local sublattices randomly distributed in the system. One sublattice has sites occupied by $S_{i}^{z}=0.0$ and the other sublattice has sites occupied randomly by $S_{i}^{z}= \pm 1$. Thus, when $r<-1.0$, the description of the two interpenetrating sublattice model seems to be required for this model, although the new phase (often called the staggered quadrupolar phase) corresponds to the phase IV with $m=0.0$ and $q \neq 0.0$ in Fig. 3 obtained from the magnetization process of the single sublattice model.

\section{Higher spin problems}

In the previous sections, we have studied spin- $1 / 2$ and spin- 1 Ising models by means of the differential operator technique based on the identities. Now, how can the technique be extended to the Ising models with spin values higher than $S=1$ ? For example, from Eq. (12) we can also obtain the following form:

$$
\left\langle\left\{f_{i}\right\} S_{i}^{z}\right\rangle=\left.S\left\langle\left\{f_{i}\right\} \exp \left(E_{i} \nabla\right)\right\rangle B_{S}(\beta x)\right|_{x=0} .
$$

Then, in order to treat the exponential operator, the identities (20) and (92) have been used in spin-1/2 and spin-1 Ising models. These identities are often called the Van der Waerden identities. For $S=3 / 2$, on the other hand, the Van der Waerden identity is given by

$$
\exp \left(a S_{i}^{z}\right)=A(a)+B(a) S_{i}^{z}+C(a)\left(S_{i}^{z}\right)^{2}+D(a)\left(S_{i}^{z}\right)^{3}
$$

with 


$$
\begin{aligned}
& A(a)=\frac{1}{8}[9 \cosh (a / 2)-\cosh (3 a / 2)], \\
& B(a)=\frac{1}{12}[27 \sinh (a / 2)-\sinh (3 a / 2)], \\
& C(a)=\frac{1}{2}[\cosh (3 a / 2)-\cosh (a / 2)], \\
& D(a)=\frac{1}{3}[\sinh (3 a / 2)-3 \sinh (a / 2)] .
\end{aligned}
$$

In general, for an arbitrary spin value larger than $S=1$, the following transformation is valid:

$$
\exp \left(a S_{i}^{z}\right)=\sum_{n=0}^{2 S} A_{n}(a)\left(S_{i}^{z}\right)^{n} .
$$

Substituting $S_{i}^{z}=-S, \ldots,+S$ into (129), one can get a set of $(2 S+1)$ parameters for the spin variables as well as the coefficients $A_{n}(a)$, like (128) [30].

By selecting the spin- $S$ BC model, let us discuss in this section how the differential operator technique can be extended to the Ising problem with a higher spin value $(S \geq 1)$. The Hamiltonian is also defined by (86). Then, the spin operator $S_{i}^{z}$ in $(86)$ can take $(2 S+1)$ values allowed for a spin $S$, namely $S_{i}^{z}=-S$, $-S+1, \ldots, S-1, S$. For the spin- $S$ BC model, one can derive the following identities:

$$
\left\langle\left\{f_{i}\right\} S_{i}^{z}\right\rangle=\left\langle\left\{f_{i}\right\} F_{S}\left(E_{i}\right)\right\rangle=\left.\left\langle\left\{f_{i}\right\} \exp \left(E_{i} \nabla\right)\right\rangle F_{S}(x)\right|_{x=0}
$$

and

$$
\left\langle\left(S_{i}^{z}\right)^{2}\right\rangle=\left\langle G_{S}\left(E_{i}\right)\right\rangle=\left.\left\langle\exp \left(E_{i} \nabla\right)\right\rangle G_{S}(x)\right|_{x=0},
$$

where $E_{i}=J \sum_{j} S_{j}^{z}$ and the functions $F_{S}(x)$ and $G_{S}(x)$ depend on the value of $S$. For $S=1, F_{S}(x)$ and $G_{S}(x)$ are given by (89) and (97). They are defined by

$$
\begin{aligned}
& F_{S}(x)=\frac{1}{2} \frac{3 \sinh (3 \beta x / 2)+\exp (-2 D \beta) \sinh (\beta x / 2)}{\cosh (3 \beta x / 2)+\exp (-2 D \beta) \cosh (\beta x / 2)}, \\
& G_{S}(x)=\frac{1}{4} \frac{9 \cosh (3 \beta x / 2)+\exp (-2 D \beta) \cosh (\beta x / 2)}{\cosh (3 \beta x / 2)+\exp (-2 D \beta) \cosh (\beta x / 2)}
\end{aligned}
$$

for $S=3 / 2$,

$$
\begin{aligned}
& F_{S}(x)=\frac{4 \sinh (2 \beta x)+2 \exp (-3 D \beta) \sinh (\beta x)}{2 \cosh (2 \beta x)+2 \exp (-3 D \beta) \cosh (\beta x)+\exp (-4 D \beta)}, \\
& G_{S}(x)=\frac{8 \cosh (2 \beta x)+2 \exp (-3 D \beta) \cosh (\beta x)}{2 \cosh (2 \beta x)+2 \exp (-3 D \beta) \cosh (\beta x)+\exp (-4 D \beta)}
\end{aligned}
$$

for $S=2$, and so on.

Now, using the Van der Waerden identity (129) for an arbitrary spin $S$, the exponential operator in (130) and (131) can be exactly represented as the product of the sum of $(2 S+1)$ independent variables at each neighboring site. In contrast to the spin- $1 / 2$ and spin- 1 cases, it is not so easy to treat these Ising spin identities in the simple forms. In fact, even when the simplest decoupling approximation, 
like (94), is adopted for different neighboring sites, one must introduce the $2 S$ independent spin variables

$$
m=\left\langle S_{i}^{z}\right\rangle, \quad q=\left\langle\left(S_{i}^{z}\right)^{2}\right\rangle, \quad r=\left\langle\left(S_{i}^{z}\right)^{3}\right\rangle, \ldots, \quad v=\left\langle\left(S_{i}^{z}\right)^{2 S}\right\rangle
$$

for the evaluation of the identities. Furthermore, expanding the right-hand side of these identities, the calculations of the coefficients in the polynomials of the $2 S$ order parameter become too complicated forms of the coefficients, $A_{n}(a)$, in the Van der Waerden identity (129), like (128) for $S=3 / 2$.

In order to avoid such calculation difficulties inherent to the usage of the exact spin- $S$ Van der Waerden identity, Kaneyoshi et al. [30] have introduced the generalized but approximated Van der Waerden identity

$$
\exp \left(a S_{i}^{z}\right)=\cosh (\eta a)+\frac{S_{i}^{z}}{\eta} \sinh (\eta a)
$$

with

$$
\eta=\left\langle\left(S_{i}^{z}\right)^{2}\right\rangle
$$

which is valid for any spin value $S$. In particular, for $S=1 / 2$ Eq. (135) reduces to the exact one

$$
\exp \left(a S_{i}^{z}\right)=\cosh (a / 2)+2 S_{i}^{z} \sinh (a / 2)
$$

since the parameter $\eta$ is then given by $\eta=1 / 2$. In fact, the framework based on (135) has given reasonable results for various physical quantities in comparison with those based on the exact Van der Waerden identities [31]. Thus, Eqs. (130) and (131) can be transformed, by the use of (135), into the forms

$$
\left\langle\left\{f_{i}\right\} S_{i}^{z}\right\rangle=\left.\left\langle\left\{f_{i}\right\} \prod_{j}\left[\cosh (J \eta \nabla)+\frac{S_{j}^{z}}{\eta} \sinh (J \eta \nabla)\right]\right\rangle F_{S}(x)\right|_{x=0}
$$

and

$$
\left\langle\left(S_{i}^{z}\right)^{2}\right\rangle=\left.\left\langle\prod_{j}\left[\cosh (J \eta \nabla)+\frac{S_{j}^{z}}{\eta} \sinh (J \eta \nabla)\right]\right\rangle G_{S}(x)\right|_{x=0} .
$$

By the use of these formulations, one can evaluate various magnetic properties of the spin- $S$ BC model, as discussed in the previous sections. At this place, we shall discuss only the phase diagram of the system. In the same way as that of Sec. 6.1, one can obtain

$$
m^{2}=\frac{1-\bar{a}}{\bar{b}}
$$

in the vicinity of the second-order phase transition line. The general expressions of the parameters $\bar{a}$ and $\bar{b}$ are given in [30].

From the general framework, the phase diagram can be evaluated numerically. In this case, the obtained result must be distinguished, depending on whether $S$ is an integer or a half-integer:

(i) For an integer spin $(S=1,2, \ldots)$, there always exists a point at which the tricritical condition $(\bar{a}=1$ and $\bar{b}=0)$ is satisfied, when the value of $D / J$ takes a negative number. The phase diagram is given by the same form as Fig. 3 for the spin-one BC model. Within the present formulation, the tricritical point for the 
spin-one BC model is also given by (112). On the other hand, the tricritical point for $S=2$ can be determined as

$$
\frac{D_{\mathrm{t}}}{z J}=-0.498 \text { for } S=2 \text {. }
$$

With the increase in $S$, therefore, the value of $D_{t} / z J$ seems to approach to the critical value $D_{\mathrm{t}} / z J=-0.5$.

(ii) For a half-integer spin $(S=3 / 2,5 / 2, \ldots)$, the parameter $\bar{b}$ is always negative for any $z$. In other words, the BC model with a half-integer spin does not show the tricritical behavior. This is reasonable, since the system does not include the $S_{i}^{z}=0$ state. The phase diagram is schematically presented by Fig. 4 . In fact,

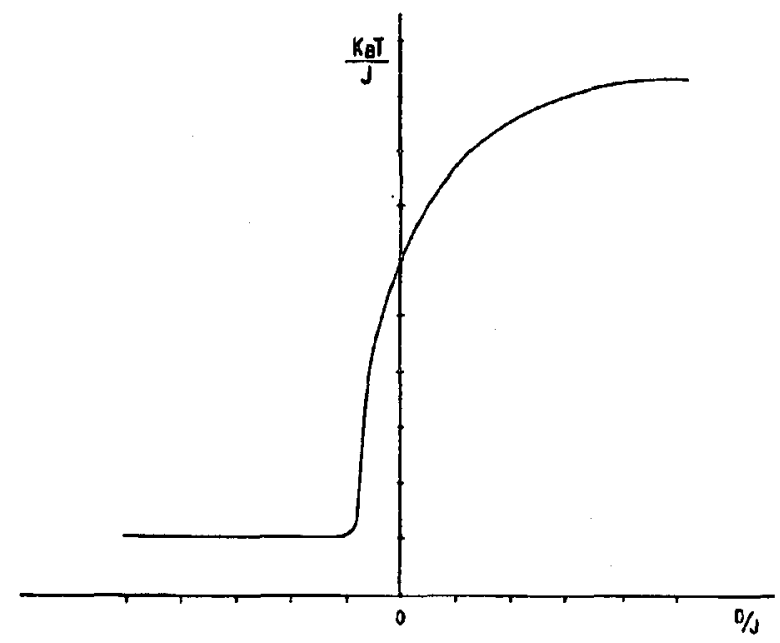

Fig. 4. Phase diagram of the BC model with a half-integer spin $(S>1 / 2)$.

when $D \rightarrow \infty$, the functions $F_{S}(x)$ and $G_{S}(x)$ are respectively given by

$$
F_{S}(x)=S \tanh (S \beta x) \text { and } G_{S}(x)=S^{2} \text {, }
$$

which means that only the $S_{i}^{z}= \pm S$ state is allowed in the limit. Substituting (142) into $\bar{a}$ and noticing that $\eta=S$, the condition $\bar{a}=1$ determining $T_{\mathrm{c}}$ is given, within the present formulation, by

$$
1=\left.z \sinh (S J \nabla) \cosh ^{z-1}(S J \nabla) \tanh \left(\beta_{c} S x\right)\right|_{x=0} .
$$

On the other hand, when $D \rightarrow-\infty$, the functions $F_{S}(x)$ and $G_{S}(x)$ are given by

$$
F_{S}(x)=\frac{1}{2} \tanh \left(\frac{1}{2} \beta x\right) \text { and } G_{S}(x)=\frac{1}{4}
$$

which implies that only the $S_{i}^{z}= \pm 1 / 2$ state is allowed in the limit. Here, the transition temperature is determined from

$$
1=\left.z \sinh \left(\frac{J}{2} \nabla\right) \cosh ^{z-1}\left(\frac{J}{2} \nabla\right) \tanh \left(\frac{\beta_{c}}{2} x\right)\right|_{x=0},
$$

which is nothing but the Zernike equation (33) for $S=1 / 2$. Thus, for $|D| \rightarrow \infty$, the phase diagram of the $\mathrm{BC}$ model with a half-integer spin may approach to the 
two constant values continuously, namely one - the transition temperature of the $S_{i}^{z}= \pm S$ state and the other - the transition temperature of the $S_{i}^{z}= \pm 1 / 2$ state.

\section{Spin-S transverse Ising model}

The Ising model with a transverse field $\Omega$ is described by the Hamiltonian

$$
H=-\frac{1}{2} \sum_{i, j} J_{i j} S_{i}^{z} S_{j}^{z}-\Omega \sum_{i} S_{i}^{x},
$$

where the $S_{i}^{z}, S_{i}^{x}$ are the components of quantum spin $S$ operators and the first summation is over all nearest-neighbor pairs.

The transverse Ising model for the case $S=1 / 2$ has been extensively investigated for many years by the use of various techniques. In fact, the model is useful for the study of cooperative phenomena and phase transitions in many systems including order-disorder ferroelectrics, induced moment ferromagnets, and cooperative Jahn-Teller systems. In this section, let us discuss how the present technique can be extended to the model with an arbitrary spin $S$.

For the application of the differential operator technique to an Ising spin system, it is necessary to find the spin correlation identity. As discussed in Sec. 2, it is always based on the fundamental fact that $H_{i}$ and $H^{\prime}$ commute each other, when the Hamiltonian is separated into two parts $H_{i}$ and $H^{\prime}$ like (4). For the transverse Ising model, on the other hand, one should notice that $H_{i}$ and $H^{\prime}$ do not commute when the Hamiltonian (146) is separated into two parts. Owing to the fact, the exact identities cannot be derived for the transverse Ising model. As proposed by SáBarreto et al. [32], however, the approximated spin correlation relations can be derived for the Hamiltonian (146) and they have been used as the starting formula.

Let us start at first from the derivation of the approximated identities. The expectation value including the longitudinal or transverse spin operator at a site $i$ is given by

$$
\left\langle\left\{f_{i}\right\} S_{i}^{\alpha}\right\rangle=\frac{T_{r}\left[\left\{f_{i}\right\} S_{i}^{\alpha} \exp (-\beta H)\right]}{T_{r}[\exp (-\beta H)]},
$$

where $\alpha=z$ or $x$. Separating the Hamiltonian (146) into two parts, $H_{i}$ is given by

$$
H_{i}=-E_{i} S_{i}^{z}-\Omega S_{i}^{x}
$$

with

$$
E_{i}=\sum_{j} J_{i j} S_{j}^{z} .
$$

By noticing that $H_{i}$ and $H^{\prime}$ do not commute, we can obtain the following result for the expectation value (147):

$$
\begin{aligned}
& \left\langle\left\{f_{i}\right\} S_{i}^{\alpha}\right\rangle=\left\langle\left\{f_{i}\right\} \frac{T_{r\{i\}} S_{i}^{\alpha} \exp \left(-\beta H_{i}\right)}{T_{r\{i\}} \exp \left(-\beta H_{i}\right)}\right\rangle \\
& -\left\langle\left\{f_{i}\right\}\left[\frac{T_{r} S_{i}^{\alpha} \exp \left(-\beta H_{i}\right)}{T_{r\{i\}} \exp \left(-\beta H_{i}\right)}-S_{i}^{\alpha}\right] \bar{\Delta}\right\rangle
\end{aligned}
$$


with

$$
\bar{\Delta}=1-\exp \left(-\beta H_{i}\right) \exp \left(-\beta H^{\prime}\right) \exp \left[\beta\left(H_{i}+H^{\prime}\right)\right],
$$

where $T_{r\{i\}}$ means the partial trace with respect to the lattice site $i$.

Equation (150) is also an exact relation, although it is difficult to deal with owing to the presence of the second thermal average. Accordingly, let us introduce an approximation as follows:

$$
\begin{aligned}
& \left\langle\left\{f_{i}\right\}\left[\frac{T_{r\{i\}} S_{i}^{\alpha} \exp \left(-\beta H_{i}\right)}{T_{r\{i\}} \exp \left(-\beta H_{i}\right)}-S_{i}^{\alpha}\right] \bar{\Delta}\right\rangle \\
& \approx\left\langle\left\{f_{i}\right\}\left[\frac{T_{r\{i\}} S_{i}^{\alpha} \exp \left(-\beta H_{i}\right)}{T_{r\{i\}} \exp \left(-\beta H_{i}\right)}-S_{i}^{\alpha}\right]\right\rangle\langle\bar{\Delta}\rangle,
\end{aligned}
$$

from which we can obtain a relation

$$
\left\langle\left\{f_{i}\right\} S_{i}^{\alpha}\right\rangle=\left\langle\left\{f_{i}\right\} \frac{T_{r\{i\}} S_{i}^{\alpha} \exp \left(-\beta H_{i}\right)}{T_{r\{i\}} \exp \left(-\beta H_{i}\right)}\right\rangle \text {. }
$$

Then, the decoupling (152) can be viewed as a zeroth-order approximation of the exact relation. In other words, Eq. (153) can be assumed to be obtained from the approximation

$$
\left\langle\left\{f_{i}\right\}\left(\bar{S}_{i}^{\alpha}-S_{i}^{\alpha}\right)\right\rangle=0,
$$

where

$$
\bar{S}_{i}^{\alpha}=\frac{T_{r\{i\}} S_{i}^{\alpha} \exp \left(-\beta H_{i}\right)}{T_{r\{i\}} \exp \left(-\beta H_{i}\right)} .
$$

The approximated relation (153) has been used by many authors as the starting point for the statistics of the transverse Ising model. In fact, it has been successfully applied to a number of interesting physical systems for $S=1 / 2$ and $S=1$ [33]. In order to calculate the relation (153), one must diagonalize the form of $H_{i}$ by the use of the rotational transformation

with

$$
\begin{gathered}
S_{i}^{z}=S_{i}^{z^{\prime}} \cos \phi_{i}-S_{i}^{x^{\prime}} \sin \phi_{i}, \\
S_{i}^{x}=S_{i}^{z^{\prime}} \sin \phi_{i}+S_{i}^{x^{\prime}} \cos \phi_{i}
\end{gathered}
$$

$$
\cos \phi_{i}=E_{i} / \theta_{i}, \quad \sin \phi_{i}=\Omega / \theta_{i},
$$

where $\theta_{i}$ is defined by

$$
\phi_{i}=\left(\Omega^{2}+E_{i}^{2}\right)^{1 / 2} \text {. }
$$

For the evaluation of (153), furthermore, one must use the following matrices:

$$
\begin{aligned}
& \left\langle\sigma\left|S_{i}^{z^{\prime}}\right| \sigma^{\prime}\right\rangle=\delta_{\sigma, \sigma^{\prime}}, \\
& \left\langle\sigma\left|S_{i}^{x}\right| \sigma\right\rangle=\left\langle\sigma^{\prime}\left|S_{i}^{x^{\prime}}\right| \sigma\right\rangle=\frac{1}{2} \delta_{\sigma^{\prime}, \sigma-1}[(S+\sigma)(S-\sigma+1)]^{\frac{1}{2}},
\end{aligned}
$$

where $\delta$ is the Kronecker $\delta$-function and $\sigma$ can take $2 S+1$ values allowed for a spin $S$, namely $\sigma=-S,-S+1, \ldots, S-1, S$. From these procedures, one can get the relations [34]:

$$
\left\langle\left\{f_{i}\right\} S_{i}^{z}\right\rangle=\left\langle\left\{f_{i}\right\} \frac{E_{i}}{\theta_{i}} f_{S}\left(E_{i}\right)\right\rangle,
$$




$$
\left\langle\left\{f_{i}\right\} S_{i}^{x}\right\rangle=\left\langle\left\{f_{i}\right\} \frac{\Omega}{\theta_{i}} f_{S}\left(E_{i}\right)\right\rangle,
$$

where the function $f_{S}(x)$ depends on the value of $S$. The function is given by

$$
\begin{aligned}
& f_{S}(x)=\frac{1}{2} \tanh \left(\frac{1}{2} \beta y\right) \quad \text { for } \quad S=\frac{1}{2}, \\
& f_{S}(x)=\frac{2 \sinh (\beta y)}{2 \cosh (\beta y)+1} \text { for } S=1,
\end{aligned}
$$

and so on, where the parameter $y$ is defined by

$$
y=\left(\Omega^{2}+x^{2}\right)^{1 / 2} \text {. }
$$

In the limit of $\Omega=0,\left\langle\left\{f_{i}\right\} S_{i}^{x}\right\rangle=0$ and $\theta_{i}=E_{i}$, so that for $S=1 / 2 \mathrm{Eq}$. (160) reduces to the Callen identity (10) for the spin-1/2 Ising model. In particular, the standard MFA is given by replacing the operator $E_{i}$ in (160) and (161) with the a veraged value $\left\langle E_{i}\right\rangle$; by setting $\left\{f_{i}\right\}=1$ into them, the longitudinal and transverse magnetizations of the MFA are given by

$$
\begin{aligned}
& \left\langle S_{i}^{z}\right\rangle=\frac{\left\langle E_{i}\right\rangle}{\bar{\theta}_{i}} f_{S}\left(\left\langle E_{i}\right\rangle\right), \\
& \left\langle S_{i}^{x}\right\rangle=\frac{\Omega}{\bar{\theta}_{i}} f_{S}\left(\left\langle E_{i}\right\rangle\right),
\end{aligned}
$$

with

$$
\bar{\theta}_{i}=\left(\Omega^{2}+\left(\left\langle E_{i}\right\rangle\right)^{2}\right)^{1 / 2} .
$$

Thus, the relations (160) and (161), although they are approximately derived, are expected to give fairly nice results especially for small values of $\Omega$.

By introducing the differential operator technique, the magnetizations can be rewritten as

$$
\begin{aligned}
& \left\langle S_{i}^{z}\right\rangle=\left.\left\langle\exp \left(E_{i} \nabla\right)\right\rangle F_{S}(x)\right|_{x=0}, \\
& \left\langle S_{i}^{x}\right\rangle=\left.\left\langle\exp \left(E_{i} \nabla\right)\right\rangle H_{S}(x)\right|_{x=0},
\end{aligned}
$$

where the functions $F_{S}(x)$ and $H_{S}(x)$ are defined by

$$
\begin{aligned}
& F_{S}(x)=\frac{x}{\left(\Omega^{2}+x^{2}\right)^{1 / 2}} f_{S}(x), \\
& H_{S}(x)=\frac{\Omega}{\left(\Omega^{2}+x^{2}\right)^{1 / 2}} f_{S}(x) .
\end{aligned}
$$

In order to treat the exponential operator in (168) and (169) for an arbitrary spin $S$, let us use the generalized but approximated Van der Waerden identity (135). Then, for a spin $S$ higher than $S=1 / 2$ one has to evaluate the parameter $\eta$ defined by (136). It can be derived in the same way as $\left\langle S_{i}^{z}\right\rangle$ and $\left\langle S_{i}^{x}\right\rangle$ by the use of (135):

$$
\begin{aligned}
& \eta^{2}=\left\langle\left(S_{i}^{z}\right)^{2}\right\rangle=\left.\left\langle\exp \left(E_{i} \nabla\right)\right\rangle G_{S}(x)\right|_{x=0} \\
& =\left.\left\langle\prod_{j}\left[\cosh \left(J_{i j} \eta \nabla\right)+\frac{S_{j}^{z}}{\eta} \sinh \left(J_{i j} \eta \nabla\right)\right]\right\rangle G_{S}(x)\right|_{x=0},
\end{aligned}
$$


where the function $G_{S}(x)$ also depends on the spin value $S$. When $S=1$, for example, it is given by

$$
G_{S}(x)=\frac{\Omega^{2}+\left(x^{2}+y^{2}\right) \cosh (\beta y)}{y^{2}[1+2 \cosh (\beta y)]}
$$

where $y$ is defined by (164).

As discussed in the previous sections, various magnetic properties of the spin- $S$ transverse Ising model can be derived from these relations. IIowever, further development becomes untractable and consequently we have to introduce some approximation. Then, the simplest approximation is also to introduce the decoupling approximation, like (94). Using the decoupling approximation and taking account of the fact that $J_{i j}$ is given by $J$ for nearest neighbors, the longitudinal and transverse magnetizations as well as the parameter $\eta$ are given by

$$
\begin{aligned}
& m_{z}=\left\langle S_{i}^{z}\right\rangle=\left.\left[\cosh (J \eta \nabla)+\frac{m_{z}}{\eta} \sinh (J \eta \nabla)\right]^{z} F_{S}(x)\right|_{x=0} \\
& m_{x}=\left\langle S_{i}^{x}\right\rangle=\left.\left[\cosh (J \eta \nabla)+\frac{m_{z}}{\eta} \sinh (J \eta \nabla)\right]^{z} H_{S}(x)\right|_{x=0}
\end{aligned}
$$

and

$$
\eta^{2}=\left\langle\left(S_{i}^{z}\right)^{2}\right\rangle=\left.\left[\cosh (J \eta \nabla)+\frac{m_{z}}{\eta} \sinh (J \eta \nabla)\right]^{z} G_{S}(x)\right|_{x=0}
$$

where $z$ is the coordination number.

Now, in order to investigate the phase diagram of a spin- $S$ transverse Ising model, one must notice the following facts. In a finite transverse field, the $S_{i}^{z}$ component of the system is disordered at high temperatures, but below a transition temperature $T_{\mathrm{c}}$ it orders, so that $m_{z} \neq 0.0$ and the direction of the moment changes continuously, although there is an order with $m_{x} \neq 0.0$ at all temperatures. From Eqs. (173) and (174), therefore, the second-order phase transition line is determined by solving the coupled equations

$$
\begin{aligned}
& 1=\left.z \frac{1}{\eta} \sinh (J \eta \nabla) \cosh ^{z-1}(J \eta \nabla) F_{S}(x)\right|_{x=0}, \\
& \eta^{2}=\left.\cosh ^{z}(J \eta \nabla) G_{S}(x)\right|_{x=0} .
\end{aligned}
$$

In particular, for $S=1 / 2$ the parameter $\eta$ is given by $\eta=1 / 2$, so that when $\Omega=0.0 \mathrm{Eq}$. (176) reduces to (145), which is nothing but that for the spin-1/2 Ising model in the Zernike approximation.

At this place, let us show some typical results especially for the spin-1/2 transverse Ising model, in order to compare them with those obtained from the MFA, the high-temperature series expansion (SE) and the renormalization-group (RG) methods [35]. Figure 5 shows the variation of $T_{\mathrm{c}}$ versus $\Omega$ in the spin-1/2 transverse Ising model on the square lattice $(z=4)$. The critical temperature $T_{c}$ gradually decreases from its Ising value at $\Omega=0.0$ and rapidly vanishes when the transverse field approaches some critical value $\Omega_{c}$. For comparison, the critical values of $\Omega_{\mathrm{c}}$ obtained from such methods as well as the present framework (EFT) are collected in Table IV. On the other hand, the thermal behaviors of the longitudinal and transverse magnetizations in the system are given in Fig. 6 by selecting the two values of $\Omega(\Omega=0.1 J$ and $\Omega=0.3 J)$. The results clearly express that 


\section{TABLE IV}

Critical values of $\Omega$ for spin- $1 / 2$

transverse Ising model on square

lattice.

\begin{tabular}{l|c|c|c|c}
\hline \hline & MFA & EFT & SE & RG \\
\hline$\Omega_{\mathrm{c}} / J$ & 2.0 & 1.37 & 1.52 & 0.77
\end{tabular}

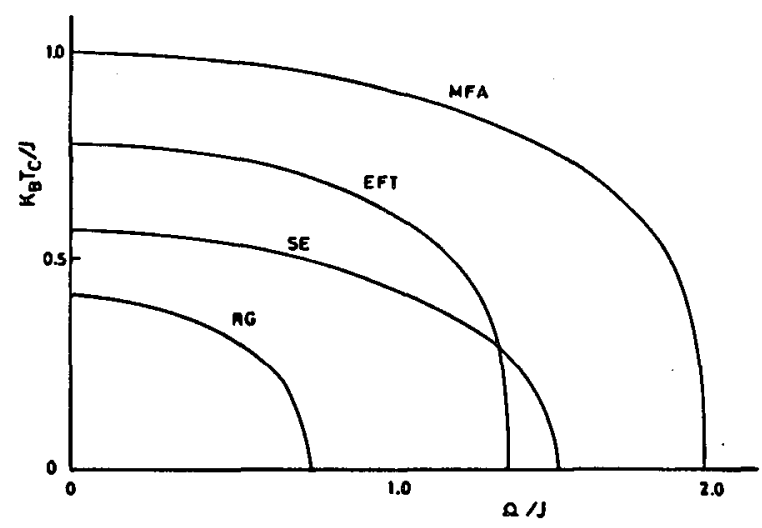

Fig. 5. Transition temperature for the spin- $1 / 2$ transverse Ising model on square latlice plotted as a function of $\Omega$.

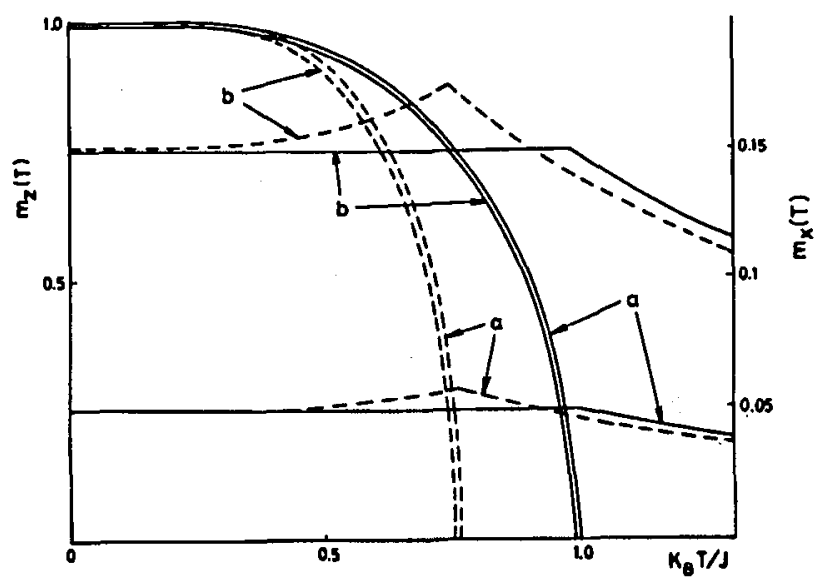

Fig. 6. Thermal dependencies of longitudinal and transverse magnetizations ( $m_{z}$ and $m_{x}$ ) for the ferromagnetic square lattice with (a) $\Omega=0.1 \mathrm{~J}$ and (b) $\Omega=0.3 \mathrm{~J}$. The solid lines are the results of the MFA and the dashed lines are the results of the EFT. 
the larger the transverse field, the smaller the longitudinal magnetization; the role of the transverse field is essentially to inhibit the ordering of the $S_{i}^{z}$ component. Furthermore, it is worth to compare the EFT results with those of the MFA. The MFA results are plotted in the figure as the dashed lines. The present results for $m_{x}$ are qualitatively different from the MFA behavior; below the transition temperature of $m_{z}$ the transverse magnetization $m_{x}$ is very sensitive to temperature, in contrast with the constant behavior predicted by the MFA, namely (166). Similar behavior is also reported in the thermal variation of $m_{x}$ obtained from the Monte Carlo calculations for the special case of $S=\infty$ [36].

Finally, one should notice that the effects of transverse field on the critical temperature, the longitudinal and transverse magnetizations in the higher spin transverse Ising systems are very similar to those of the spin- $1 / 2$ transverse Ising model. In particular, the critical value $\Omega_{c}$ may increase with the increasing spin value $S$.

\section{Concluding remarks}

In the present work, by making use of exact (or approximate) spin relations and taking advantage of the differential operator expansion technique, various Ising spin systems have been discussed, although the Potts model has not been included (see [37]). We have seen that the differential operator technique affords us the opportunity of obtaining fairly reliable information concerning such systems, even when the simplest decoupling approximation is used for the treatment of multispin correlation functions. While the formulations given in this work are applicable to the Ising spin systems without any disorder, it has been also clarified that they can be successfully applied to disordered spin systems. In a disordered spin system, magnetic atoms are usually frozen at their fixed positions, where a certain disorder can be included at each site or bond. For such a quenched disorder system, the thermally averaged quantity $\langle X\rangle$ is a functional of some set of disorder included in it. That is to say, it is assumed to calculate the spin sum (since the system is assumed to be in thermal equilibrium) and then to average the quantity over some disorder parameters. In other words, we assume that any correlation between spin and random averages is absent in the system. Mathematically, the physical quantity $X$ in a disordered system can be expressed as

$$
\left\langle\langle X\rangle_{r}=\int\langle X\rangle_{\{R\}} P(\{R\}) \mathrm{d}\{R\},\right.
$$

where $\langle\cdots\rangle_{r}$ refers to the random average over all possible configurations of the random parameters $\{R\}$ and $P(\{R\})$ is the probability distribution function.

In a spin-1/2 Ising system with random bonds, for example, the atomic magnetization based on the decoupling approximation (30) can be then rewritten in the form [38]:

$$
m_{i}=\left\langle\left\langle\mu_{i}\right\rangle\right\rangle_{r}=\left.\prod_{j}\left[\left\langle\cosh \left(J_{i j} \nabla\right)\right\rangle_{r}+m_{j}\left\langle\sinh \left(J_{i j} \nabla\right)\right\rangle_{r}\right] \tanh (\beta x+h)\right|_{x=0} .
$$

IIere, $(\cdots\rangle_{r}$ denotes the random bond average defined by

$$
\left\langle\langle X\rangle_{r}=\int\langle X\rangle_{\left\{J_{i, j}\right\}} \prod_{i<j} P\left(J_{i, j}\right) \prod_{i<j} \mathrm{~d} J_{i j} .\right.
$$


In particular, when $H=0.0$ and $P\left(J_{i j}\right)$ is given by

$$
P\left(J_{i j}\right)=p \delta\left(J_{i j}-J\right)+(1-p) \delta\left(J_{i j}\right)
$$

for nearest-neighbor sites, it corresponds to the standard bond dilution problem. The averaged magnetization of the bond-diluted spin-1/2 Ising ferromagnet is given by

$$
m=\left.\{1-p+p[\cosh (J \nabla)+m \sinh (J \nabla)]\}^{z} \tanh (\beta x)\right|_{x=0} .
$$

Thus, the critical temperature $T_{\mathrm{c}}$ can be determined by solving

$$
1=\left.z p \sinh (J \nabla)[1-p+p \cosh (J \nabla)]^{z-1} \tanh \left(\beta_{\mathrm{c}} x\right)\right|_{x=0} .
$$

The critical temperature $T_{\mathrm{c}}$ depends on $p$, decreases with the decrease in $p$ and reduces to zero at a critical concentration $p_{\mathrm{B}}^{*}$ of the bond percolation (for example, $p_{\mathrm{B}}^{*}=0.4284$ for $z=4$ ). As noted in Sec. 4.1, the result is just that which is obtained if the Matsudaira first-order approximation is used for bond dilution. The fact also includes that the formulation is superior to the standard MFA with $p_{\mathrm{B}}^{*}=0.0$.

On the other hand, the same equation as (183) can be derived even for the site dilution problem, when the decoupling (or the Matsudaira) approximation is used [39]. It means that the critical concentration $p_{S}^{*}$ of the site percolation is equivalent to the value of $p_{\mathrm{B}}^{*}$. Various methods noted in Sec. 4.1, which are essentially equivalent to the decoupling approximation (30), have yielded unsatisfactory results $\left(p_{\mathrm{B}}^{*}=p_{\mathrm{S}}^{*}\right)$ for the percolation limit, since the exact calculations for bond and site diluted spin-1/2 Ising systems prove the relation of $p_{\mathrm{B}}^{*}<p_{\mathrm{S}}^{*}$. Thus, an improvement in the configurational averaging, beyond the simplest decoupling approximation, seems to be necessary for the bond-diluted model, in order to get a correct percolation limit.

The differential operator technique has also been applied to study various situations in disordered spin systems, including spin-one Ising systems with random bond and crystal-field interactions. In particular, the spin-one Ising model with a random crystal-field interaction should be referred here, since some outstanding results may be obtained in going from the MFA to more sophisticated theories [40]. The Hamiltonian of the model is defined, instead of the BC model (86), by

$$
H=-J \sum_{i, j} S_{i}^{z} S_{j}^{z}+\sum_{i} D_{i}\left(S_{i}^{z}\right)^{2},
$$

where $D_{i}$ is a random crystal-field constant distributed according to a probability distribution function $P\left(D_{i}\right)$, such as

$$
P\left(D_{i}\right)=p \delta\left(D_{i}-D\right)+(1-p) \delta\left(D_{i}\right) .
$$

Within the framework of the MFA, several authors have examined the phase diagram of the model and predicted some fascinating phenomena, especially for large values of $p$ [41]. However, these results are different from those based on the EFT any do not provide any insight into the nature of possible phase transition lines. The reason is as follows. When $D=\infty$ (or $D$ is larger than $D_{c}$ ), $N p$ spins among the total number $N$ of spins are in the $S_{i}^{z}=0$ state and $N(1-p)$ spins behave as the usual Ising spins with $D=0.0$. Therefore, the spins in the $S_{i}^{z}=0$ state simply correspond to the introduction of $N p$ non-magnetic atoms in the 
system, since they are inactive as regards producing ferromagnetic ordering. The situation is very similar to that of dilution problem. Thus, in order to treat the model system (184), a theory better than the MFA is required. In particular, the re-entrant phenomenon due to the crystal-field disorder has been predicted by the use of the EFT [40].

As reviewed in this work, the differential operator technique in Ising spin systems has a simple mathematical structure. Through some sort of successive approximation scheme, the effects of correlations can explicitly and systematically be included. This procedure, without introducing mathematical complexities, provides results which are quite superior to those obtained within the standard MFA. As noted in Sec. 7, the method can be extended to an Ising problem with a higher spin value.

In particular, the magnetization of a ferromagnetic system can be expressed in a polynomial form, which is well illustrated by employing the simplest decoupling approximation, such as (30). Because of the fact, the formulations in Sec. 4 have been applied to study many other interesting problems in surface magnetism, thin films and multilayers which are now current topics of magnetism [42]. Here, notice that these works are discussed on the basis of spin-1/2 Ising model and its variants, such as the Mills model in surface magnetism [43]. On the other hand, recent experiments show that there exists a very strong anisotropy field acting on spins in the surfaces of thin films. The role of surface anisotropy has been proved to be of paramount importance in determining the magnetic properties of surfaces and thin films [44]. In magnetic multilayers, detailed analyses of the experimental data reveal that the interfaces normally exist and they are constructed by some mixed layers with a random distribution of the two types of magnetic atoms consisting of the multilayer. Therefore, the standard theoretical models which treat magnetic multilayers consisting of two spin- $1 / 2$ ferromagnetic layers with different bulk properties should also be modified, in order to include the effects of the disordered interfaces. The influences of the surface anisotropy in surface magnetism and thin films as well as the disordered interfaces in magnetic multilayers on their magnetic properties can be treated by the use of formulations given in Secs. 4-8 [45].

\section{Acknowledgment}

This review comes out in collaboration with many co-workers. Especially, the author wishes to thank I.P. Fittipaldi for many enlightening and useful discussions as well as for sending the preprint which was very useful in writing Sec. 4.3.

\section{References}

[1] H.B. Callen, Phys. Letl. 4, 161 (1963).

[2] M. Suzuki, Phys. Lett. 19, 267 (1965).

[3] N. Matsudaira, J. Phys. Soc. Jpn. 35, 1593 (1973).

[4] R. Honmura, T. Kaneyoshi, J. Phys. C 12, 3979 (1979). 
[5] F.C. SáBarreto, I.P. Fittipaldi, Rev. Bras. Fis. 11, 745 (1981).

[6] T. Kaneyoshi, H. Beyer, J. Phys. Soc. Jpn. 49, 1306 (1980).

[7] F. Zernike, Physica 7, 565 (1940).

[8] T. Kaneyoshi, Phys. Lett. A 76, 67 (1980).

[9] J. Mielnicki, T. Balcerzak, V.H. Truong, G. Wiatrowski, L. Wojtczak, J. Magn. Magn. Mater. 58, 325 (1986); T. Balcerzak, J. Magn. Magn. Mater. 97, 152 (1991).

[10] N. Boccara, Phys. Lett. A 94, 185 (1983).

[11] A. Benyoussef, N. Boccara, J. Phys. (France) 44, 1143 (1983); A. Benyoussef, N. Boccara, M. Saber, J. Phys. C 18, 4275 (1985).

[12] M.E. Lines, Phys. Rev. B 9, 3927 (1974).

[13] L. Onsager, J. Am. Chem. Soc. 58, 1486 (1936).

[14] T.H. Berlin, M. Kac, Phys. Rev. 86, 821 (1952).

[15] T. Kaneyoshi, I.P. Fittipaldi, R. Honmura, T. Manabe, Phys. Rev. B 24, 481 (1981); T. Kaneyoshi, I. Tamura, Phys. Rev. B 25, 4679 (1982); R. Honmura, Phys. Rev. $B$ 30, 348 (1984).

[16] L. Onsager, Phys. Rev. 65.197 (1944); M.E. Fisher, Rep. Prog. Phys. 30, 615 (1967).

[17] A. Bobak, M. Jaščur, Phys. Slalus Solidi B 135, K9 (1986); P. Tomczak, E.F. Sarmento, A.F. Siqueira, A.R. Ferchmin, Phys. Slatus Solidi B 142, 551 (1987).

[18] J.O. Indekeu, A. Maritan, A.L. Stella, J. Plıys. A 15, L291 (1982); Phys. Rev. B 35,305 (1987).

[19] I.P. Fittipaldi, D.F. DeAbuquerque, J. Magn. Magn. Maler. 104-107, 236 (1992);

I.P. Fittipaldi, unpublished work, 1992.

[20] T. Kaneyoshi, I.P. Fittipaldi, II. Beyer, Phys. Stalus Solidi B 102, 393 (1980).

[21] M. Blume, Phys. Rev. 141, 517 (1966); H.W. Capel, Physica 32, 966 (1966).

[22] M. Blume, V.J. Emery, R.B. Griffiths, Phys. Rev. A 4, 1071 (1971).

[23] I. Tamura, T. Kaneyoshi, Prog. Theor. Phys. 66, 1892 (1981).

[24] A.F. Siqueira, I.P. Fittipaldi, Physica A 138, 592 (1986).

[25] T. Kaneyoshi, J. Phys. C 19, L557 (1986).

[26] I.P. Fittipaldi, T. Kaneyoshi, J. Phys., Condens. Maller 1, 6513 (1989).

[27] J.W. Tucker, J. Magn. Magn. Maler. 80, 203 (1989).

[28] T. Kaneyoshi, J. Magn. Magn. Mater. 98, 185 (1991).

[29] Y.L. Wang, C. Wentworth, J. Appl. Phys. 61, 411 (1987).

[30] T. Kaneyoshi, J.W. Tucker, M. Jaščur, Physica A 186, 495 (1992).

[31] T. Kaneyoshi, M. Jaščur, Physica B 179, 317 (1992); Plıys. Rev. B 46, 3374 (1992).

[32] F.C. SáBarreto, I.P. Fittipaldi, B. Zeks, Ferroelectrics 39, 1103 (1981); F.C. SaBarreto, I.P. Fittipaldi, Physica A 129, 360 (1985).

[33] E.F. Sarmento, I. Tamura, L.E.M.C. de Oliveira, T. Kaneyoshi, J. Phys. C 17, 3195 (1984); I. Tamura, E.F. Sarmento, T. Kaneyoshi, J. Phys. C 17, 3207 (1984); T. Kaneyoshi, E.F. Sarmento, I.P. Fittipaldi, Phys. Rev. B 38, 2649 (1988); I.P. Fittipaldi, E.F. Sarmento, T. Kaneyoshi, Physica A 186, 591 (1992).

[34] T. Kaneyoshi, M. Jaščur, I.P. Fittipaldi, unpublished work, 1992. 
[35] R.J. Elliot, C. Wood, J. Phys. C 4, 2359 (1971); R.R. dos Santos, J. Phys. C 15, 3141 (1981); J. Phys. A 14, L179 (1981).

[36] P. Prelovsek, I. Sega, J. Phys. C 11, 2103 (1978).

[37] R. Honmura, E.F. Sarmento, C. Tsallis, I.P. Fittipaldi, Phys. Rev. B 29, 2761 (1984).

[38] R. Honmura, A. Khater, I.P. Fittipaldi, T. Kaneyoshi, Solid Slale Commun. 41, 385 (1982); E.F.'Sarmento, C. Tsallis, Phys. Rev. B 27, 5784 (1983).

[39] T. Kaneyoshi, I. Tamura, E.F. Sarmento, Phys. Rev. B 28, 6491 (1983).

[40] T. Kaneyoshi, J. Mielnicki, J. Phys., Condens. Matter 2, 8773 (1990); T. Kaneyoshi, Phys. Status Solidi B 170, 313 (1992).

[41] A. Benyoussef, T. Biaz, M. Saber, M. Touzani, J. Phys. C 20, 5349 (1987); C.E.I. Carneiro, V.B. Henriques, R.A. Salinas, J. Phys., Condens. Matter 1, 571 (1989); N. Boccara, A. Elkenz, M. Saber, J. Phys., Condens. Matter 1, 5721 (1989).

[42] T. Kaneyoshi, Rev. Solid State Sci. 2, 39 (1988).

[43] D.L. Mills, Phys. Rev. B 3, 3887 (1971).

[44] T. Kaneyoshi, J. Phys., Condens. Matter 3, 4497 (1991).

[45] T. Kaneyoshi, M. Jaščur, J. Magn. Magn. Mater. 118, 17 (1993); A. Khater, G. LeGal, T. Kaneyoshi, Phys. Lett. A 171, 237 (1992); T. Kaneyoshi, T. Balcerzak, unpublished work, 1992. 